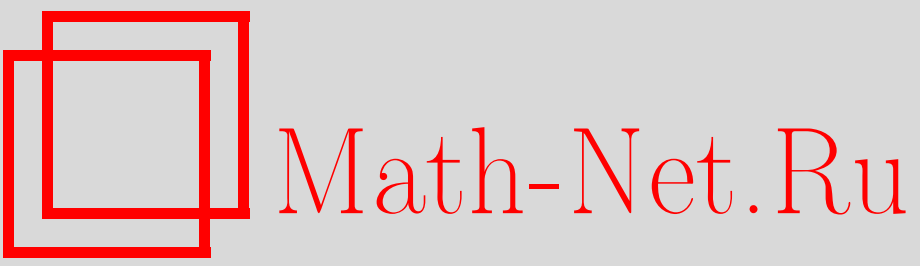

Д. А. Закора, Представление решений одного интегро-дифференциального уравнения и приложения, Итоги науки и техн. Сер. Соврем. мат. и ее прил. Темат. обз., 2019, том 171, 78-93

DOI: https://doi.org/10.36535/0233-6723-2019-171-78-93

Использование Общероссийского математического портала Math-Net.Ru подразумевает, что вы прочитали и согласны с пользовательским соглашением

http://www.mathnet.ru/rus/agreement

Параметры загрузки:

IP : 54.197 .217 .227

26 апреля 2023 г., 04:51:46 
УДК 517.929, 517.984.52

\title{
ПРЕДСТАВЛЕНИЕ РЕШЕНИЙ \\ ОДНОГО ИНТЕГРО-ДИФФЕРЕНЦИАЛЬНОГО УРАВНЕНИЯ И ПРИЛОЖЕНИЯ
}

\author{
(c) 2019 г. $\quad$ Д. А. ЗАКОРА
}

\begin{abstract}
АннотАция. В работе рассмотрено интегро-дифференциальное уравнение второго порядка с неограниченными операторными коэффициентами в гильбертовом пространстве, представляющее собой абстрактное гиперболическое уравнение, возмущенное интегральным слагаемым с ядром разностного типа специального вида. Это уравнение возникает при описании различных вязкоупругих систем. По системе корневых элементов операторного пучка, ассоциированного с рассматриваемым уравнением, построен $p$-базис в ортогональной сумме гильбертовых пространств. С использованием этого базиса найдено представление решения исследуемого уравнения. Приведены приложения к задачам из теории вязкоупругости.
\end{abstract}

Ключевые слова: интегро-дифференциальное уравнение, спектральный анализ, операторный пучок, $p$-базис.

\section{REPRESENTATION OF SOLUTIONS OF A CERTAIN INTEGRO-DIFFERENTIAL EQUATION AND APPLICATIONS}

\author{
(c) 2019 D. A. ZAKORA
}

\begin{abstract}
In this paper, we consider a second-order integro-differential equation with unbounded operator coefficients in a Hilbert space, which is an abstract hyperbolic equation perturbed by an integral term with a difference-type kernel of a special form. This equation arises in the description of various viscoelastic systems. Using the system of generalized eigenvectors of the operator pencil associated with the equation considered, we construct a $p$-basis in the orthogonal sum of Hilbert spaces. Using this basis, we find a representation of the solution of the equation. We also discuss possible applications to problems of viscoelasticity.
\end{abstract}

Keywords and phrases: integro-differential equation, spectral analysis, operator bundle, $p$-base.

AMS Subject Classification: 45J05, 45C05

1. Постановка задачи. Пусть $H$ - сепарабельное гильбертово пространство, $A$ - самосопряженный положительно определенный оператор, действующий в гильбертовом пространстве $H$ и имеющий обратный компактный $\left(A^{-1} \in \mathfrak{S}_{\infty}(H)\right)$. Рассмотрим следующую задачу Коши:

$$
\frac{d^{2} u(t)}{d t^{2}}=-\alpha_{0} A u(t)+\sum_{l=1}^{m} \int_{0}^{t} e^{-b_{l}(t-s)} \alpha_{-l} A u(s) d s+f(t), \quad u(0)=u^{0}, \quad u^{\prime}(0)=u^{1},
$$

Работа выполнена при финансовой поддержке Министерства образования и науки Российской федерации (проект № 14.Z50.31.0037). 
где $0=: b_{0}<b_{1}<b_{2}<\ldots<b_{m}, \alpha_{-l}>0(l=\overline{0, m})$; при этом

$$
\alpha_{0}-\sum_{l=1}^{m} \alpha_{-l} b_{l}^{-1}>0
$$

Уравнение из (1) описывает различные системы из теории вязкоупругости, например, колебания вязкоупругого тела, занимающего ограниченную область, продольные колебания однородного вязкоупругого стержня, поперечные колебания неоднородной по толщине вязкоупругой балки или пластины, поперечные колебания ортотропной пластинки из гомогенного полимера, армированного упругими нитями или пленками и т. д. (см. [3, гл. 6, § 23]). Уравнение Гуртина-Пипкина (см. [11]), описывающее процесс распространения тепла в средах с памятью с конечной скоростью и с ядром специального вида, может быть также записано в виде уравнения из (1).

В [8] впервые было установлено убывание к нулю решений однородного уравнения типа (1) с ростом времени, однако без оценки скорости. В дальнейшем были найдены различные условия на ядра, обеспечивающие экспоненциальную или полиномиальную устойчивость нулевого решения однородного уравнения типа (1). Приведенные выше условия на числовые коэффициенты обеспечивают (см., например, [7]) экспоненциальную устойчивость уравнения из (1).

В $[2,10]$ проводится спектральный анализ уравнения из (1) в случае бесконечного количества экспоненциальных слагаемых, получены утверждения о локализации и асимптотике спектра. В [14] (см. также [1, гл. 3, § 3.2, п. 3.2.2]) получено представление решения рассматриваемого уравнения в виде суммы слагаемых, отвечающих точкам спектра символа интегро-дифференциального уравнения (операторного пучка, ассоциированного с уравнением). При этом предполагается, что спектр оператора $A$ простой. Доказательство основано на исследовании спектра символа интегро-дифференциального уравнения и использовании теоремы Пэли-Винера.

В настоящей работе спектр оператора $A$ не предполагается простым, однако ядро в уравнении из (1) содержит лишь конечное количество слагаемых. Доказательство основано на сведении (1) к задаче Коши для дифференциально операторного уравнения первого порядка в ортогональной сумме гильбертовых пространств $\mathcal{H}=\bigoplus_{l=1}^{m+2} H$, последующем исследовании и использовании системы корневых элементов главного оператора.

\section{2. Вывод основного операторного уравнения.}

Определение 1. Сильным решением задачи Коши (1) назовем такую функцию $u(t)$, что $u(t) \in \mathcal{D}(A), u^{\prime}(t) \in \mathcal{D}\left(A^{1 / 2}\right)$ при $t \in \mathbb{R}_{+}=[0 ;+\infty), A u(t), A^{1 / 2} u^{\prime}(t) \in C\left(\mathbb{R}_{+} ; H\right), u(t) \in C^{2}\left(\mathbb{R}_{+} ; H\right)$, выполнены начальные условия и уравнение из (1) для любого $t \in \mathbb{R}_{+}$.

Утверждение о разрешимости задачи Коши (1) может быть получено с использованием утверждений из $[9,12]$ или $[13$, гл. $11, \S 6$, п. 4].

Теорема 1. Пусть $u^{0} \in \mathcal{D}(A), u^{1} \in \mathcal{D}\left(A^{1 / 2}\right), f(t) \in C^{1}\left(\mathbb{R}_{+} ; H\right)$. Тогда задача Коши (1) имеет единственное сильное решение.

Предположим, что задача $(1)$ имеет единственное сильное решение $u(t)$ и $u^{0} \in \mathcal{D}(A)$. Сведем $(1)$ к задаче Коши для дифференциально-операторного уравнения первого порядка. Из того факта, что $A^{1 / 2} u^{\prime}(t), A^{1 / 2} u(t) \in C\left(\mathbb{R}_{+} ; H\right)$, следует (см. [4, гл. $3, \S 1$, п. 1]), что $\left(A^{1 / 2} u(t)\right)^{\prime}=A^{1 / 2} u^{\prime}(t)$, и мы можем при каждом $l=\overline{1, m}$ провести интегрирование по частям:

$$
\int_{0}^{t} e^{-b_{l}(t-s)} b_{l} A^{1 / 2} u(s) d s=A^{1 / 2} u(t)-e^{-b_{l} t} A^{1 / 2} u^{0}-\int_{0}^{t} e^{-b_{l}(t-s)} A^{1 / 2} u^{\prime}(s) d s .
$$


С помощью этого соотношения преобразуем уравнение из (1):

$$
\begin{aligned}
& \frac{d^{2} u(t)}{d t^{2}}=-A^{1 / 2}\left[\beta_{0} A^{1 / 2} u(t)+\sum_{l=1}^{m} \int_{0}^{t} e^{-b_{l}(t-s)} \beta_{l} A^{1 / 2} \frac{d u(s)}{d s} d s\right]+f_{0}(t), \quad u(0)=u^{0}, \quad u^{\prime}(0)=u^{1}, \\
& f_{0}(t):=f(t)-\sum_{l=1}^{m} \frac{\alpha_{-l}}{b_{l}} e^{-b_{l} t} A u^{0}, \quad \beta_{0}:=\alpha_{0}-\sum_{l=1}^{m} \frac{\alpha_{-l}}{b_{l}}>0, \quad \beta_{l}:=\frac{\alpha_{-l}}{b_{l}}>0 \quad(l=\overline{1, m}) .
\end{aligned}
$$

Введем для функции $u(t)$ объекты

$$
v(t):=u^{\prime}(t), \quad v_{0}(t):=\beta_{0}^{1 / 2} A^{1 / 2} u(t), \quad v_{l}(t):=\int_{0}^{t} e^{-b_{l}(t-s)} \beta_{l}^{1 / 2} A^{1 / 2} \frac{d u(s)}{d s} d s, \quad l=\overline{1, m},
$$

и перепишем уравнение из (2), а также продифференцированные соотношения из (3) в виде следующей системы уравнений:

$$
\left\{\begin{array}{l}
\frac{d v(t)}{d t}=-A^{1 / 2}\left[\beta_{0}^{1 / 2} v_{0}(t)+\sum_{l=1}^{m} \beta_{l}^{1 / 2} v_{l}(t)\right]+f_{0}(t), \\
\frac{d v_{0}(t)}{d t}=-\left[-\beta_{0}^{1 / 2} A^{1 / 2} v(t)\right], \\
\frac{d v_{l}(t)}{d t}=-\left[-\beta_{l}^{1 / 2} A^{1 / 2} v(t)+b_{l} v_{l}(t)\right], \quad l=\overline{1, m} .
\end{array}\right.
$$

Эту систему (с начальными условиями) будем трактовать как задачу Коши для дифференциально операторного уравнения первого порядка в гильбертовом пространстве $\mathcal{H}:=\bigoplus_{l=1}^{m+2} H$ :

$$
\frac{d \xi}{d t}=-\mathcal{A} \xi+\mathcal{F}(t), \quad \xi(0)=\xi^{0}
$$

где

$$
\begin{gathered}
\xi(t):=(v(t) ; w(t))^{\tau}=\left(v(t) ; v_{0}(t) ; v_{1}(t) ; \ldots ; v_{m}(t)\right)^{\tau}, \\
\xi^{0}:=\left(u^{1} ; \beta_{0}^{1 / 2} A^{1 / 2} u^{0} ; 0 ; \ldots ; 0\right)^{\tau}, \quad \mathcal{F}(t):=\left(f_{0}(t) ; 0 ; \ldots ; 0\right)^{\tau} .
\end{gathered}
$$

Для оператора $\mathcal{A}$ имеют место следующие формулы:

$$
\begin{gathered}
\mathcal{A}=\left(\begin{array}{cc}
0 & A^{1 / 2} \mathcal{Q}^{*} \\
-\mathcal{Q} A^{1 / 2} & \mathcal{G}^{\prime}
\end{array}\right), \quad \mathcal{Q}:=\left(\beta_{0}^{1 / 2}, \beta_{1}^{1 / 2}, \ldots, \beta_{m}^{1 / 2}\right)^{\tau}, \quad \mathcal{G}:=\operatorname{diag}\left(0, b_{1}, \ldots, b_{m}\right), \\
\mathcal{D}(\mathcal{A})=\left\{\xi=(v ; w)^{\tau} \in \mathcal{H} \mid v \in \mathcal{D}\left(A^{1 / 2}\right), \mathcal{Q}^{*} w=\sum_{l=0}^{m} \beta_{l}^{1 / 2} v_{l} \in \mathcal{D}\left(A^{1 / 2}\right)\right\},
\end{gathered}
$$

где символ $\tau$ означает операцию транспонирования.

Замечание 1 . Можно показать, что оператор $-\mathcal{A}$ является генератором $C_{0}$-полугруппы; таким образом, если выполнены условия теоремы 1 , то задача (4) имеет единственное решение $\xi(t) \in C^{1}\left(\mathbb{R}_{+} ; \mathcal{H}\right) \cap C\left(\mathbb{R}_{+} ; \mathcal{D}(\mathcal{A})\right)$. Используя $(3)$, из $\xi(t)$ можно восстановить решение $u(t)$ задачи Коши (1).

3. О пересчете корневых элементов оператора $\mathcal{A}$ и ассоциированного с ним пучка. Рассмотрим задачу о спектре оператора $\mathcal{A}$ :

$$
\mathcal{A} \xi=\lambda \xi, \quad \xi \in \mathcal{D}(\mathcal{A}) \subset \mathcal{H},
$$

где $\lambda \in \mathbb{C}$-спектральный параметр, $\xi$-амплитудный элемент.

При $\lambda \notin\left\{0, b_{1}, \ldots, b_{m}\right\}=\sigma(\mathcal{G})$ свяжем с (6) спектральную задачу

$$
L(\lambda) u:=\left[-\lambda A^{-1}+\mathcal{Q}^{*}(\mathcal{G}-\lambda)^{-1} \mathcal{Q}\right] u=\left[-\lambda A^{-1}-\frac{1}{\lambda} \beta_{0}+\sum_{l=1}^{m} \frac{1}{b_{l}-\lambda} \beta_{l}\right] u=0, \quad u \in H .
$$


Замечание 2. Непосредственно проверяется, что $\left\{0, b_{1}, \ldots, b_{m}\right\} \notin \sigma(\mathcal{A})$ и

$$
\mathcal{A}-\lambda=\operatorname{diag}\left(A^{1 / 2}, \mathcal{I}\right)\left(\begin{array}{cc}
I & \mathcal{Q}^{*}(\mathcal{G}-\lambda)^{-1} \\
0 & \mathcal{I}
\end{array}\right)\left(\begin{array}{cc}
L(\lambda) & 0 \\
0 & \mathcal{G}-\lambda
\end{array}\right)\left(\begin{array}{cc}
I & 0 \\
-(\mathcal{G}-\lambda)^{-1} \mathcal{Q} & \mathcal{I}
\end{array}\right) \operatorname{diag}\left(A^{1 / 2}, \mathcal{I}\right),
$$

при $\lambda \notin\left\{0, b_{1}, \ldots, b_{m}\right\}$, где $\mathcal{I}$ - единичный оператор. Отсюда следует, что $\sigma(\mathcal{A})=\sigma(L(\lambda))$.

Для дальнейших рассуждений нам понадобятся вспомогательные леммы о связи цепочки из собственного и присоединенного к нему элементов пучка $\mathcal{A}(\lambda)$ с некоторой функцией из $\mathcal{H}$ и о связи цепочек элементов некоторых специальных оператор-функций.

Определение 2 (см. [6, гл. $2, \S 11$, с. 61]). Пусть $\lambda_{0}$-собственное значение, а $\eta_{0}$-отвечающий ему собственный элемент оператор-функции $\mathcal{A}(\lambda) \in \mathcal{L}(\mathcal{H})$, т.е. $\mathcal{A}\left(\lambda_{0}\right) \eta_{0}=0$. Элементы $\eta_{1}, \eta_{2}, \ldots, \eta_{n-1}$ называют присоединенными $к$ собственному элементу $\eta_{0}$, если

$$
\sum_{k=0}^{j}(k !)^{-1} \mathcal{A}^{(k)}\left(\lambda_{0}\right) \eta_{j-k}=0, \quad j=1,2, \ldots, n-1 .
$$

Число $n$ называют длиной цепочки $\left\{\eta_{k}\right\}_{k=0}^{n-1}$ из собственного и присоединенных элементов.

Лемма 1 (см. [6, гл. $2, \S 11$, лемма 11.3]). Элементы $\eta_{0}, \eta_{1}, \ldots, \eta_{n-1}$ образуют иепочку из собственного и присоединенных элементов $\mathcal{A}(\lambda)$, отвечающуюо числу $\lambda_{0}$, тогда и только тогда, когда существует функиия $\eta(\lambda)$, голоморфная в некоторой окрестности точки $\lambda_{0}$, такая, что $\eta\left(\lambda_{0}\right) \neq 0, \eta^{(k)}\left(\lambda_{0}\right)=k ! \eta_{k}(k=0,1, \ldots, n-1)$ и функиия $\mathcal{A}(\lambda) \eta(\lambda)$ имеет в точке $\lambda_{0}$ нуль кратности, большей или равной $n$.

Определение 3 (см. [6, гл. $2, \S 11$, с. 62]). Пусть $\eta(\lambda)$ - функция из $\mathcal{H}$, причем $\eta\left(\lambda_{0}\right) \neq 0$ и $\mathcal{A}\left(\lambda_{0}\right) \eta\left(\lambda_{0}\right)=0$. Если порядок нуля функции $\mathcal{A}(\lambda) \eta(\lambda)$ в точке $\lambda_{0}$ равен $n$, то $\eta(\lambda)$ называется производящей функиией для цепочки из собственного и присоединенных элементов $\left\{(k !)^{-1} \eta^{(k)}\left(\lambda_{0}\right)\right\}_{k=0}^{n-1}$ оператор-функции $\mathcal{A}(\lambda)$. Число $n$ будем называть рангом производящей функции $\eta(\lambda)$.

Рассмотрим операторный пучок $\mathcal{A}(\lambda)$, действующий в гильбертовом пространстве $\mathcal{H}=H \oplus \mathcal{H}_{0}$, и соответствующую спектральную задачу:

$$
\mathcal{A}(\lambda) \eta:=\left(\begin{array}{ll}
\mathcal{A}_{11}(\lambda) & \mathcal{A}_{12}(\lambda) \\
\mathcal{A}_{21}(\lambda) & \mathcal{A}_{22}(\lambda)
\end{array}\right)\left(\begin{array}{c}
v \\
w
\end{array}\right)=\left(\begin{array}{l}
0 \\
0
\end{array}\right), \quad(v ; w)^{\tau} \in \mathcal{H}=H \oplus \mathcal{H}_{0}
$$

С задачей (8), при $\lambda \notin \sigma\left(\mathcal{A}_{22}(\lambda)\right)$, свяжем спектральную задачу

$$
L(\lambda) v=\left[\mathcal{A}_{11}(\lambda)-\mathcal{A}_{12}(\lambda) \mathcal{A}_{22}^{-1}(\lambda) \mathcal{A}_{21}(\lambda)\right] v=0, \quad v \in H .
$$

Замечание 3. В области $\lambda \in \mathbb{C} \backslash \sigma\left(\mathcal{A}_{22}(\lambda)\right)$ спектральную задачу (8) можно переписать следующим образом:

$$
\mathcal{A}(\lambda) \eta=\left(\begin{array}{cc}
I & \mathcal{A}_{12}(\lambda) \mathcal{A}_{22}^{-1}(\lambda) \\
0 & \mathcal{I}
\end{array}\right)\left(\begin{array}{cc}
L(\lambda) & 0 \\
0 & \mathcal{A}_{22}(\lambda)
\end{array}\right)\left(\begin{array}{cc}
I & 0 \\
\mathcal{A}_{22}^{-1}(\lambda) \mathcal{A}_{21}(\lambda) & \mathcal{I}
\end{array}\right)\left(\begin{array}{l}
v \\
w
\end{array}\right)=\left(\begin{array}{l}
0 \\
0
\end{array}\right) .
$$

Отсюда после замены $\left(v ; \mathcal{A}_{22}^{-1}(\lambda) \mathcal{A}_{21}(\lambda) v+w\right)^{\tau}=:\left(v ; w_{v}\right)^{\tau}$ найдем, что в области $\lambda \in \mathbb{C} \backslash \sigma\left(\mathcal{A}_{22}(\lambda)\right)$ спектральные задачи (8) и (9) эквивалентны.

Лемма 2. Пусть $\lambda \notin \sigma\left(\mathcal{A}_{22}(\lambda)\right)$. Функиия $\eta(\lambda):=(v(\lambda) ; w(\lambda))^{\tau}$ из $\mathcal{H}$ является производящей функиией ранга п пучка $\mathcal{A}(\lambda)$ в точке $\lambda_{0} \notin \sigma\left(\mathcal{A}_{22}(\lambda)\right)$ тогда и только тогда, когда $v(\lambda)$ является производящей функиией ранга п пучка $L(\lambda)$ в точке $\lambda_{0} u$

$$
w(\lambda)=-\mathcal{A}_{22}^{-1}(\lambda) \mathcal{A}_{21}(\lambda) v(\lambda)+\left(\lambda-\lambda_{0}\right)^{n} \mathcal{A}_{22}^{-1}(\lambda) p(\lambda),
$$

где $p(\lambda)$ - функиия, голоморфная в некоторой окрестности точки $\lambda_{0}$. 
Доказательство. Доказательство этой леммы следует рассуждениям [6, гл. 2, § 12, лемма 12.3]. Начнем с достаточности. Пусть $v(\lambda)$ является производящей функцией ранга $n$ пучка $L(\lambda)$ в точке $\lambda_{0} \notin \sigma\left(\mathcal{A}_{22}(\lambda)\right)$ и выполнено соотношение $(10)$. Поскольку $L(\lambda) v(\lambda)$ имеет в точке $\lambda_{0}$ нуль кратности $\geqslant n$, то из вида $L(\lambda)$ получим:

$$
L(\lambda) v(\lambda)=\left[\mathcal{A}_{11}(\lambda)-\mathcal{A}_{12}(\lambda) \mathcal{A}_{22}^{-1}(\lambda) \mathcal{A}_{21}(\lambda)\right] v(\lambda)=\left(\lambda-\lambda_{0}\right)^{n} q(\lambda)
$$

где $q(\lambda)$ - некоторая функция, голоморфная в окрестности точки $\lambda_{0}$. Подставим (10) в (11) и запишем полученное соотношение вместе с (10) в виде одного векторно-матричного выражения в $\mathcal{H}$; после простых преобразований получим

$$
\mathcal{A}(\lambda)(v(\lambda) ; w(\lambda))^{\tau}=\left(\lambda-\lambda_{0}\right)^{n}\left(q(\lambda)+\mathcal{A}_{12}(\lambda) \mathcal{A}_{22}^{-1}(\lambda) p(\lambda) ; p(\lambda)\right)^{\tau}
$$

Отсюда следует, что $\eta(\lambda)=(v(\lambda) ; w(\lambda))^{\tau}$ есть производящая функция ранга $n$ пучка $\mathcal{A}(\lambda)$ в точке $\lambda_{0}$. Достаточность доказана.

Пусть теперь функция $\eta(\lambda)=(v(\lambda) ; w(\lambda))^{\tau}$ является производящей функцией ранга $n$ пучка $\mathcal{A}(\lambda)$ в точке $\lambda_{0}$. По условию теоремы $\mathcal{A}(\lambda) \eta(\lambda)$ имеет в точке $\lambda_{0}$ нуль кратности $\geqslant n$; следовательно,

$$
\begin{aligned}
& \mathcal{A}_{11}(\lambda) v(\lambda)+\mathcal{A}_{12}(\lambda) w(\lambda)=\left(\lambda-\lambda_{0}\right)^{n} r(\lambda), \\
& \mathcal{A}_{21}(\lambda) v(\lambda)+\mathcal{A}_{22}(\lambda) w(\lambda)=\left(\lambda-\lambda_{0}\right)^{n} p(\lambda),
\end{aligned}
$$

где $r(\lambda), p(\lambda)$ - некоторые функции, голоморфные в окрестности точки $\lambda_{0}$. Из (13) следует (10). Подставив (10) в (12) получим, что

$$
L(\lambda) v(\lambda)=\left(\lambda-\lambda_{0}\right)^{n}\left(r(\lambda)-\mathcal{A}_{12}(\lambda) \mathcal{A}_{22}^{-1}(\lambda) p(\lambda)\right) .
$$

Отсюда следует, что $L(\lambda) v(\lambda)$ имеет в точке $\lambda_{0}$ нуль порядка не ниже $n$. Лемма доказана.

В качестве следствия из леммы 2 получим следующую лемму о пересчете корневых элементов спектральных задач (8) и (9).

Лемма 3. Пусть набор элементов $\left\{\eta_{k}=\left(v_{k} ; w_{k}\right)^{\tau}\right\}_{k=0}^{n-1}$ является цепочкой из собственного и присоединенных элементов задачи (8), отвечающей собственному значению $\lambda_{0}\left(\lambda_{0} \notin \sigma\left(\mathcal{A}_{22}(\lambda)\right)\right)$; тогда $\left\{v_{k}\right\}_{k=0}^{n-1}-$ чепочка из собственного и присоединенных элементов задачи (9), отвечающая собственному значению $\lambda_{0}$.

Обратно, пусть набор элементов $\left\{v_{k}\right\}_{k=0}^{n-1}-$ чепочка из собственного и присоединенных элементов спектральной задачи (9), отвечающая собственному значению $\lambda_{0}$; тогда $\left\{\eta_{k}=\left(v_{k} ; w_{k}\right)^{\tau}\right\}_{k=0}^{n-1}$, где

$$
w_{k}=-\left.\sum_{l=0}^{k} \frac{1}{(k-l) !} \frac{d^{k-l}}{d \lambda^{k-l}} \mathcal{A}_{22}^{-1}(\lambda) \mathcal{A}_{21}(\lambda)\right|_{\lambda=\lambda_{0}} v_{l}, \quad k=\overline{0, n-1}
$$

- чепочка из собственного и присоединенных элементов спектральной задачи (8).

Доказательство. По лемме 2 , если функция $\eta(\lambda)=(v(\lambda) ; w(\lambda))^{\tau}$ из $\mathcal{H}$ является производящей функцией ранга $n$ пучка $\mathcal{A}(\lambda)$ в точке $\lambda_{0} \notin \sigma\left(\mathcal{A}_{22}(\lambda)\right)$, то функция $v(\lambda)$ является производящей функцией ранга $n$ пучка $L(\lambda)$ в точке $\lambda_{0}$. Отсюда и из леммы 1 следует прямое утверждение.

Обратно, пусть функция $v(\lambda)$ является производящей функцией из $H$ ранга $n$ пучка $L(\lambda)$ в точке $\lambda_{0}$. Определим функцию $w(\lambda)$ по формуле (10). Тогда по лемме 2 функция $\eta(\lambda)=(v(\lambda) ; w(\lambda))^{\tau}$ 
из $\mathcal{H}$ является производящей функцией ранга $n$ пучка $\mathcal{A}(\lambda)$ в точке $\lambda_{0}$. При этом (см. определение 3)

$$
\begin{gathered}
w_{k}=-\left.\frac{1}{k !} \frac{d^{k}}{d \lambda^{k}}\left[\mathcal{A}_{22}^{-1}(\lambda) \mathcal{A}_{21}(\lambda) v(\lambda)+\left(\lambda-\lambda_{0}\right)^{n} \mathcal{A}_{22}^{-1}(\lambda) p(\lambda)\right]\right|_{\lambda=\lambda_{0}}= \\
=-\left.\left.\frac{1}{k !} \sum_{l=0}^{k} \frac{k !}{l !(k-l) !} \frac{d^{k-l}}{d \lambda^{k-l}} \mathcal{A}_{22}^{-1}(\lambda) \mathcal{A}_{21}(\lambda)\right|_{\lambda=\lambda_{0}} \frac{d^{l} v(\lambda)}{d \lambda^{l}}\right|_{\lambda=\lambda_{0}}= \\
=-\left.\sum_{l=0}^{k} \frac{1}{(k-l) !} \frac{d^{k-l}}{d \lambda^{k-l}} \mathcal{A}_{22}^{-1}(\lambda) \mathcal{A}_{21}(\lambda)\right|_{\lambda=\lambda_{0}} v_{l}, \quad k=\overline{0, n-1} .
\end{gathered}
$$

В качестве следствия из леммы 3 получим следующую теорему о связи собственных и присоединенных элементов оператора $\mathcal{A}$ и пучка $L(\lambda)$ (см. спектральные задачи (6) и (7) соответственно).

Теорема 2. Пусть набор элементов $\left\{\xi_{k}=\left(u_{k} ; w_{k}\right)^{\tau}\right\}_{k=0}^{n-1}$ является иепочкой из собственного и присоединенных элементов задачи (6), отвечающей собственному значению $\lambda_{0}$ $\left(\lambda_{0} \neq 0, b_{1}, \ldots, b_{m}\right) ;$ тогда набор элементов $\left\{v_{k}\right\}_{k=0}^{n-1}:=\left\{A^{1 / 2} u_{k}\right\}_{k=0}^{n-1}-$ цепочка из собственного $u$ присоединенных элементов задачи (7), отвечающая собственному значению $\lambda_{0}$.

Обратно, пусть набор элементов $\left\{v_{k}\right\}_{k=0}^{n-1}-$ цепочка из собственного и присоединенных элементов спектральной задачи (7), отвечающая собственному значению $\lambda_{0}$; тогда набор элементов $\left\{\xi_{k}=\left(A^{-1 / 2} v_{k} ; w_{k}\right)^{\tau}\right\}_{k=0}^{n-1}$, где $w_{k}=\sum_{l=0}^{k}\left(\mathcal{G}-\lambda_{0}\right)^{-(k-l+1)} \mathcal{Q} v_{l},-$ цепочка из собственного $и$ присоединенных элементов спектральной задачи (6).

Доказательство. Пусть $\lambda_{0}\left(\lambda_{0} \neq 0, b_{1}, \ldots, b_{m}\right)$ - собственное значение задачи $(6)$ и $\xi(\lambda)$ - производящая функция для цепочки $\left\{\xi_{k}:=(k !)^{-1} \xi^{(k)}\left(\lambda_{0}\right)\right\}_{k=0}^{n-1}$ из собственного и присоединенных элементов (см. определение 3). Запишем задачу (6) в виде

$$
(\mathcal{A}-\lambda) \xi=\mathcal{B} \mathcal{A}(\lambda) \mathcal{B} \xi=0, \quad \xi \in \mathcal{D}(\mathcal{A})
$$

(см. (5)), где $\mathcal{B}:=\operatorname{diag}\left(A^{1 / 2}, \mathcal{I}\right)$, а оператор-функция $\mathcal{A}(\lambda) \in \mathcal{L}(\mathcal{H})$ имеет вид

$$
\mathcal{A}(\lambda)=\left(\begin{array}{ll}
\mathcal{A}_{11}(\lambda) & \mathcal{A}_{12}(\lambda) \\
\mathcal{A}_{21}(\lambda) & \mathcal{A}_{22}(\lambda)
\end{array}\right):=\left(\begin{array}{cc}
-\lambda A^{-1} & \mathcal{Q}^{*} \\
-\mathcal{Q} & \mathcal{G}-\lambda
\end{array}\right)
$$

Отсюда следует, что $\mathcal{B} \xi(\lambda)=\left(A^{1 / 2} u(\lambda) ; w(\lambda)\right)^{\tau}$ - производящая функция для оператор-функции $\mathcal{A}(\lambda)$. Согласно лемме $2, v(\lambda):=A^{1 / 2} u(\lambda)$ - производящая функция для оператор-функции $L(\lambda)$, и первое утверждение теоремы доказано.

Пусть теперь $\lambda_{0}$ - собственное значение спектральной задачи $(7)$ и $v(\lambda)$ - производящая функция для цепочки из собственного и присоединенных элементов $\left\{v_{k}:=(k !)^{-1} v^{(k)}\left(\lambda_{0}\right)\right\}_{k=0}^{n-1}$ (см. определение 3$)$ оператор-функции $L(\lambda)$. Тогда в соответствии с леммой 3 получим, что

$$
\left(v_{k} ; w_{k}\right)^{\tau}:=\left(v_{k} ;-\left.\sum_{l=0}^{k} \frac{1}{(k-l) !} \frac{d^{k-l}}{d \lambda^{k-l}} \mathcal{A}_{22}^{-1}(\lambda) \mathcal{A}_{21}(\lambda)\right|_{\lambda=\lambda_{0}} v_{l}\right)^{\tau}, \quad k=\overline{0, n-1},
$$

- цепочка из собственного и присоединенных элементов оператор-функции $\mathcal{A}(\lambda)$, отвечающая собственному значению $\lambda_{0}$. Для вторых компонент из $(15)$ имеем, с учетом вида $\mathcal{A}_{22}(\lambda)$ и $\mathcal{A}_{21}(\lambda)$ (см. (14) и определение 3)

$$
w_{k}=\left.\sum_{l=0}^{k} \frac{1}{(k-l) !} \frac{d^{k-l}}{d \lambda^{k-l}}(\mathcal{G}-\lambda)^{-1} \mathcal{Q}\right|_{\lambda=\lambda_{0}} v_{l}=\sum_{l=0}^{k}\left(\mathcal{G}-\lambda_{0}\right)^{-(k-l+1)} \mathcal{Q} v_{l} .
$$

Таким образом, набор элементов $\left\{\eta_{k}=\left(v_{k} ; w_{k}\right)^{\tau}\right\}_{k=0}^{n-1}$ является цепочкой из собственного и присоединенных элементов оператор-функции $\mathcal{A}(\lambda)$, отвечающей собственному значению $\lambda_{0}$. Отсюда следует, что набор элементов $\left\{\xi_{k}=\left(A^{-1 / 2} v_{k} ; w_{k}\right)^{\tau}\right\}_{k=0}^{n-1}$ - цепочка из собственного и присоединенных элементов задачи (6). Теорема доказана. 
4. Теорема о спектре оператора $\mathcal{A}$. Пусть $\lambda_{k}=\lambda_{k}\left(A^{-1}\right), u_{k}=u_{k}\left(A^{-1}\right)(k \in \mathbb{N})-k$-е собственное значение и соответствующий ему нормированный к единице собственный элемент оператора $A^{-1}$ (напомним, что $\left.A^{-1} \in \mathfrak{S}_{\infty}(H)\right)$. Тогда $u_{k}$ - собственный элемент операторного пучка $L(\lambda)$, и спектр задачи (7), а значит, и спектр оператора $\mathcal{A}($ см. (5)-(6)) могут быть полностью найдены из следующей последовательности характеристических уравнений:

$$
\mathcal{Q}^{*}(\mathcal{G}-\lambda)^{-1} \mathcal{Q} \equiv-\frac{1}{\lambda} \beta_{0}+\sum_{l=1}^{m} \frac{\beta_{l}}{b_{l}-\lambda}=\lambda \lambda_{k}, \quad k \in \mathbb{N} .
$$

Здесь и далее $\mathcal{Q}, \mathcal{Q}^{*}$ и $\mathcal{G}$ будем также понимать как вектор-столбец, вектор-строку и матрицу соответственно, действующие в $\mathbb{C}^{m+1}$.

Определим характеристические функции

$$
\begin{aligned}
g_{k}(\lambda) & :=\mathcal{Q}^{*}(\mathcal{G}-\lambda)^{-1} \mathcal{Q}-\lambda \lambda_{k} \equiv-\frac{1}{\lambda} \beta_{0}+\sum_{l=1}^{m} \frac{\beta_{l}}{b_{l}-\lambda}-\lambda \lambda_{k}, \quad k \in \mathbb{N}, \\
g_{\infty}(\lambda) & :=\mathcal{Q}^{*}(\mathcal{G}-\lambda)^{-1} \mathcal{Q} \equiv-\frac{1}{\lambda} \beta_{0}+\sum_{l=1}^{m} \frac{\beta_{l}}{b_{l}-\lambda} \equiv-\frac{1}{\lambda}\left[\sum_{l=0}^{m} \beta_{l}-\sum_{l=1}^{m} \frac{\beta_{l} b_{l}}{b_{l}-\lambda}\right] .
\end{aligned}
$$

Обозначим через $\gamma_{p}(p=\overline{1, m})$ корни уравнения $g_{\infty}(\lambda)=0$. Простые геометрические рассуждения показывают, что $\gamma_{p} \in\left(b_{p-1}, b_{p}\right)\left(p=\overline{1, m}, b_{0}:=0\right), g_{\infty}^{\prime}\left(\gamma_{p}\right)>0(p=\overline{1, m})$.

Обозначим через $\lambda_{k}^{(p)}(p=\overline{1, m+2})$ корни уравнения $g_{k}(\lambda)=0$ при каждом $k \in \mathbb{N}$. Можно проверить, что это уравнение всегда имеет $m$ действительных корней $\lambda_{k}^{(p)} \in\left(\gamma_{p}, b_{p}\right)(p=\overline{1, m})$ и еще два корня. Если $g_{k}^{\prime}\left(\lambda_{k}^{(p)}\right) \neq 0(p=\overline{1, m})$, то $g_{k}^{\prime}\left(\lambda_{k}^{(p)}\right)>0$. Оставшиеся два корня $\lambda_{k}^{(m+1)}$ и $\lambda_{k}^{(m+2)}$ являются комплексно сопряженными начиная с некоторого номера $k_{0}$. В силу конечной кратности собственных значений оператора $A^{-1}$ легко видеть также, что может быть только конечное количество номеров $k \in \mathbb{N}$ при которых характеристическое уравнение $g_{k}(\lambda)=0$ имеет кратные (действительные) корни.

Применение асимптотических методов к уравнениям (16) приводит к следующей теореме.

Теорема 3. Спектр оператора $\mathcal{A}($ или пучка $L(\lambda))$ расположен в правой открытой полуплоскости и в $\mathbb{C} \backslash\left\{\gamma_{1}, \ldots, \gamma_{m}\right\}$ состоит из изолированных конечнократных собственных значений, которые расположены симметрично относительно действительной полуоси. Все собственные значения можно разбить на $(m+2)$ серии $\left\{\lambda_{k}^{(p)}\right\}_{k=1}^{\infty}(p=\overline{1, m}),\left\{\lambda_{k}^{(m+1)}\right\}_{k=1}^{\infty}:=\left\{\lambda_{k}^{(+i \infty)}\right\}_{k=1}^{\infty}$, $\left\{\lambda_{k}^{(m+2)}\right\}_{k=1}^{\infty}:=\left\{\lambda_{k}^{(-i \infty)}\right\}_{k=1}^{\infty}$ со следующим асимптотическим поведением:

$$
\begin{aligned}
& \lambda_{k}^{(p)}=\gamma_{p}+\frac{\gamma_{p}}{g_{\infty}^{\prime}\left(\gamma_{p}\right)} \lambda_{k}\left(A^{-1}\right)+O\left(\lambda_{k}^{2}\left(A^{-1}\right)\right), \quad k \rightarrow+\infty, \quad p=1,2, \ldots, m, \\
& \lambda_{k}^{( \pm i \infty)}= \pm i \alpha^{1 / 2} \lambda_{k}^{-1 / 2}\left(A^{-1}\right)+\sum_{l=1}^{m} \frac{\beta_{l} b_{l}}{2 \alpha}+O\left(\lambda_{k}^{1 / 2}\left(A^{-1}\right)\right), \quad k \rightarrow+\infty, \quad \alpha:=\sum_{l=0}^{m} \beta_{l} .
\end{aligned}
$$

5. Некоторые леммы о системах векторов в $\mathbb{C}^{m+2}$. В соответствии с теоремой 2 собственные элементы оператора $\mathcal{A}$, после группировки по сериям (см. теорему 3 ), могут быть записаны следующим образом:

$$
\begin{aligned}
\widetilde{\xi}_{k}^{(p)}=\left(A^{-1 / 2} u_{k} ;(\mathcal{G}\right. & \left.\left.-\lambda_{k}^{(p)}\right)^{-1} \mathcal{Q} u_{k}\right)^{\tau}=\left(\lambda_{k}^{1 / 2}\left(A^{-1}\right) ;\left(\mathcal{G}-\lambda_{k}^{(p)}\right)^{-1} \mathcal{Q}\right)^{\tau} u_{k} \equiv \\
& \equiv\left(\lambda_{k}^{1 / 2}\left(A^{-1}\right) ; \frac{-\beta_{0}^{1 / 2}}{\lambda_{k}^{(p)}} ; \frac{\beta_{1}^{1 / 2}}{b_{1}-\lambda_{k}^{(p)}} ; \ldots ; \frac{\beta_{m}^{1 / 2}}{b_{m}-\lambda_{k}^{(p)}}\right)^{\tau} u_{k}, \quad p=\overline{1, m+2}, \quad k \in \mathbb{N} .
\end{aligned}
$$

В связи с этой формулой докажем ряд вспомогательных утверждений. 
Лемма 4. Пусть $J:=\operatorname{diag}(1,-I)-$ матрица в $\mathbb{C}^{m+2}=\mathbb{C} \oplus \mathbb{C}^{m+1}$,

$$
\begin{gathered}
\varphi_{k}^{(p)}:=R_{k, p}\left(\lambda_{k}^{1 / 2}\left(A^{-1}\right) ;\left(\mathcal{G}-\lambda_{k}^{(p)}\right)^{-1} \mathcal{Q}\right)^{\tau}, \quad p=\overline{1, m+2}, \quad k \in \mathbb{N}, \\
R_{k, p}:= \begin{cases}{\left[g_{\infty}^{\prime}\left(\gamma_{p}\right)\right]^{-1 / 2},} & p=\overline{1, m}, \quad k \in \mathbb{N}, \\
{\left[2 \lambda_{k}\left(A^{-1}\right)\right]^{-1 / 2},} & p=m+1, m+2, \quad k \in \mathbb{N} .\end{cases}
\end{gathered}
$$

При всех $p, q=\overline{1, m+2}, k \in \mathbb{N}$ имеют место следуюшие формулы:

$$
\left.\left(J \varphi_{k}^{(p)}, \varphi_{k}^{(q)}\right)_{\mathbb{C}^{m+2}}=0, \quad \lambda_{k}^{(p)} \neq \overline{\lambda_{k}^{(q)}}\right) ; \quad\left(J \varphi_{k}^{(p)}, \overline{\varphi_{k}^{(p)}}\right)_{\mathbb{C}^{m+2}}=-g_{k}^{\prime}\left(\lambda_{k}^{(p)}\right) R_{k, p}^{2}
$$

Доказательство. При $\lambda_{k}^{(p)} \neq \overline{\lambda_{k}^{(q)}}$ из $(16),(17)$, равенства $g_{k}\left(\lambda_{k}^{(p)}\right)=0$ и тождества Гильберта находим

$$
\begin{gathered}
\left(J \varphi_{k}^{(p)}, \varphi_{k}^{(q)}\right)_{\mathbb{C}^{m+2}}=R_{k, p} R_{k, q}\left[\lambda_{k}-\left(\left(\mathcal{G}-\lambda_{k}^{(p)}\right)^{-1} \mathcal{Q},\left(\mathcal{G}-\lambda_{k}^{(q)}\right)^{-1} \mathcal{Q}\right)_{\mathbb{C}^{m+1}}\right]= \\
=R_{k, p} R_{k, q}\left[\lambda_{k}-\mathcal{Q}^{*}\left(\mathcal{G}-\overline{\lambda_{k}^{(q)}}\right)^{-1}\left(\mathcal{G}-\lambda_{k}^{(p)}\right)^{-1} \mathcal{Q}\right]= \\
=R_{k, p} R_{k, q}\left[\lambda_{k}-\frac{1}{\overline{\lambda_{k}^{(q)}}-\lambda_{k}^{(p)}}\left[\mathcal{Q}^{*}\left(\mathcal{G}-\overline{\lambda_{k}^{(q)}}\right)^{-1} \mathcal{Q}-\mathcal{Q}^{*}\left(\mathcal{G}-\lambda_{k}^{(p)}\right)^{-1} \mathcal{Q}\right]\right]= \\
=R_{k, p} R_{k, q}\left[\lambda_{k}-\frac{1}{\overline{\lambda_{k}^{(q)}}-\lambda_{k}^{(p)}}\left[\lambda_{k}^{(q)} \lambda_{k}-\lambda_{k}^{(p)} \lambda_{k}\right]\right]=0
\end{gathered}
$$

Далее, из (16) имеем

$$
\left(J \varphi_{k}^{(p)}, \overline{\varphi_{k}^{(p)}}\right)_{\mathbb{C}^{m+2}}=R_{k, p}^{2}\left[\lambda_{k}-\mathcal{Q}^{*}\left(\mathcal{G}-\lambda_{k}^{(p)}\right)^{-2} \mathcal{Q}\right]=-g_{k}^{\prime}\left(\lambda_{k}^{(p)}\right) R_{k, p}^{2}
$$

Лемма доказана.

Лемма 5. Пусть $M_{k}:=M_{k}\left(\varphi_{k}^{(1)}, \varphi_{k}^{(2)}, \ldots, \varphi_{k}^{(m+2)}\right)(k \in \mathbb{N})-$ матрица, столбцами которой являются векторы $\varphi_{k}^{(p)}(p=\overline{1, m+2})$. Имеют место следуюшие утверждения.

1. Cуществует такое $C_{1}>0$, ито $\left\|M_{k}\right\|_{\mathcal{L}\left(\mathbb{C}^{m+2}\right)} \leqslant C_{1}$ nри всех $k \in \mathbb{N}$.

2. $\operatorname{Ecлu~} g_{k}^{\prime}\left(\lambda_{k}^{(p)}\right) \neq 0(p=\overline{1, m+2}, k \in \mathbb{N})$, mo $\operatorname{det} M_{k} \neq 0$.

3. В условиях $n$. 2 существует такое $C_{2}>0$, ито $\left\|M_{k}^{-1}\right\|_{\mathcal{L}\left(\mathbb{C}^{m+2}\right)} \leqslant C_{2}$ при всех $k \in \mathbb{N}$.

Доказательство. Из теоремы 3 несложно вывести, что нормы $\left\|\varphi_{k}^{(p)}\right\|_{\mathbb{C}^{m+2}}$ равномерно ограничены по $p=\overline{1, m+2}, k \in \mathbb{N}$. Отсюда и из оценки

следует первое утверждение леммы.

$$
\left\|M_{k}\right\|_{\mathcal{L}\left(\mathbb{C}^{m+2}\right)} \leqslant\left[\sum_{p=1}^{m+2}\left\|\varphi_{k}^{(p)}\right\|_{\mathbb{C}^{m+2}}^{2}\right]^{1 / 2}
$$

Далее, с помощью формул (18), (19) из леммы 4 найдем, что

$$
M_{k}^{\tau} J M_{k}=-\operatorname{diag}\left(g_{k}^{\prime}\left(\lambda_{k}^{(1)}\right) R_{k, 1}^{2}, g_{k}^{\prime}\left(\lambda_{k}^{(2)}\right) R_{k, 2}^{2}, \ldots, g_{k}^{\prime}\left(\lambda_{k}^{(m+2)}\right) R_{k, m+2}^{2}\right) .
$$

Отсюда следует, что

$$
(-1)^{m+1}\left(\operatorname{det} M_{k}\right)^{2}=\operatorname{det} M_{k}^{\tau} J M_{k}=(-1)^{m+2} \prod_{p=1}^{m+2} g_{k}^{\prime}\left(\lambda_{k}^{(p)}\right) R_{k, p}^{2}
$$

учитывая (18) и соотношения $g_{k}^{\prime}\left(\lambda_{k}^{(p)}\right) \neq 0(p=\overline{1, m+2}, k \in \mathbb{N})$, находим

$$
\left(\operatorname{det} M_{k}\right)^{2}=-1 \cdot \frac{-g_{k}^{\prime}\left(\lambda_{k}^{(m+1)}\right)}{2 \lambda_{k}} \cdot \frac{-g_{k}^{\prime}\left(\lambda_{k}^{(m+2)}\right)}{2 \lambda_{k}} \cdot \prod_{p=1}^{m} \frac{g_{k}^{\prime}\left(\lambda_{k}^{(p)}\right)}{g_{\infty}^{\prime}\left(\gamma_{p}\right)} \neq 0 .
$$


Далее, с использованием (16), (17), теоремы 3 и (16) получаем

$$
\lim _{k \rightarrow+\infty}\left(\operatorname{det} M_{k}\right)^{2}=-\prod_{p=1}^{m} \lim _{k \rightarrow+\infty} \frac{g_{k}^{\prime}\left(\lambda_{k}^{(p)}\right)}{g_{\infty}^{\prime}\left(\gamma_{p}\right)} \cdot \lim _{k \rightarrow+\infty} \frac{-g_{k}^{\prime}\left(\lambda_{k}^{(m+1)}\right)}{2 \lambda_{k}} \cdot \lim _{k \rightarrow+\infty} \frac{-g_{k}^{\prime}\left(\lambda_{k}^{(m+2)}\right)}{2 \lambda_{k}}=-1 .
$$

Отсюда, из п. 1 и оценки

$$
\left\|M_{k}^{-1}\right\|_{\mathcal{L}\left(\mathbb{C}^{m+2}\right)} \leqslant\left|\operatorname{det} M_{k}\right|^{-1}\left\|M_{k}\right\|_{\mathcal{L}\left(\mathbb{C}^{m+2}\right)}^{m+1}
$$

(см. [5, гл. $1, \S 4$, п. 2, формула (4.12)]) следует третье утверждение в лемме. Лемма доказана.

Лемма 6. Система векторов

$$
\begin{array}{ll}
\varphi_{\infty}^{(p)}:=\left[g_{\infty}^{\prime}\left(\gamma_{p}\right)\right]^{-1 / 2}\left(0 ;\left(\mathcal{G}-\gamma_{p}\right)^{-1} \mathcal{Q}\right)^{\tau}, & p=\overline{1, m}, \\
\varphi_{\infty}^{(m+1)} \equiv \varphi_{\infty}^{(+i \infty)}:=2^{-1 / 2}\left(1 ;+i \alpha^{-1 / 2} \mathcal{Q}\right)^{\tau}, & \\
\varphi_{\infty}^{(m+2)} \equiv \varphi_{\infty}^{(-i \infty)}:=2^{-1 / 2}\left(1 ;-i \alpha^{-1 / 2} \mathcal{Q}\right)^{\tau}, & \alpha=\sum_{l=0}^{m} \beta_{l} .
\end{array}
$$

является ортонормированным базисом в $\mathbb{C}^{m+2}=\mathbb{C} \oplus \mathbb{C}^{m+1}$.

Доказательство проводится, как и в лемме 4, прямой проверкой с учетом $(16),(17), g_{\infty}\left(\gamma_{p}\right)=0$ и тождества Гильберта.

Лемма 7. Пусть $M_{\infty}:=M_{\infty}\left(\varphi_{\infty}^{(1)}, \varphi_{\infty}^{(2)}, \ldots, \varphi_{\infty}^{(m+2)}\right)$-матриц, столбиами которой являются векторы $\varphi_{\infty}^{(p)}(p=\overline{1, m+2})$. Тогда $M_{\infty}^{*}=M_{\infty}^{-1}$.

Доказательство проводится прямой проверкой с использованием леммы 6.

Замечание 4. Система (20) является предельной для системы (18) при $k \rightarrow+\infty$. Далее из системы (20) и собственных элементов оператора $A$ будет сконструирован ортонормированный базис пространства $\mathcal{H}$. Дальнейшая идея состоит в том, чтобы оценить уклонение системы собственных (а в вырожденном случае - системы корневых) элементов оператора $\mathcal{A}$ от построенного ортонормированного базиса пространства $\mathcal{H}$.

В связи с замечанием 4 докажем следующую вспомогательную лемму.

Лемма 8. Существует такое $C>0$, что при всех $k \in \mathbb{N}$

$$
\left\|M_{k}-M_{\infty}\right\|_{\mathcal{L}\left(\mathbb{C}^{m+2}\right)} \leqslant C\left[\lambda_{k}\left(A^{-1}\right)\right]^{1 / 2} .
$$

Доказательство. Как и в лемме 5 воспользуемся следующей формулой:

$$
\left\|M_{k}-M_{\infty}\right\|_{\mathcal{L}\left(\mathbb{C}^{m+2}\right)} \leqslant\left[\sum_{p=1}^{m+2}\left\|\varphi_{k}^{(p)}-\varphi_{\infty}^{(p)}\right\|_{\mathbb{C}^{m+2}}^{2}\right]^{1 / 2}
$$

Из (18), (20), теоремы 3 при $p=\overline{1, m}$ и тождества Гильберта имеем

$$
\begin{gathered}
\varphi_{k}^{(p)}-\varphi_{\infty}^{(p)}=\left[g_{\infty}^{\prime}\left(\gamma_{p}\right)\right]^{-1 / 2}\left(\lambda_{k}^{1 / 2} ;\left[\left(\mathcal{G}-\lambda_{k}^{(p)}\right)^{-1}-\left(\mathcal{G}-\gamma_{p}\right)^{-1}\right] \mathcal{Q}\right)^{\tau}= \\
=\left[g_{\infty}^{\prime}\left(\gamma_{p}\right)\right]^{-1 / 2}\left(\lambda_{k}^{1 / 2} ;\left[\lambda_{k}^{(p)}-\gamma_{p}\right]\left(\mathcal{G}-\lambda_{k}^{(p)}\right)^{-1}\left(\mathcal{G}-\gamma_{p}\right)^{-1} \mathcal{Q}\right)^{\tau}= \\
=\lambda_{k}^{1 / 2} \cdot\left[g_{\infty}^{\prime}\left(\gamma_{p}\right)\right]^{-1 / 2}\left(1 ; \lambda_{k}^{1 / 2}\left[\frac{\gamma_{p}}{g_{\infty}^{\prime}\left(\gamma_{p}\right)}+O\left(\lambda_{k}\right)\right]\left(\mathcal{G}-\lambda_{k}^{(p)}\right)^{-1}\left(\mathcal{G}-\gamma_{p}\right)^{-1} \mathcal{Q}\right)^{\tau}
\end{gathered}
$$

при $k \rightarrow+\infty$. Отсюда следует, что существует такое $C_{p}>0$, что

$$
\left\|\varphi_{k}^{(p)}-\varphi_{\infty}^{(p)}\right\|_{\mathbb{C}^{m+2}} \leqslant C_{p}\left[\lambda_{k}\left(A^{-1}\right)\right]^{1 / 2}, \quad p=\overline{1, m}, \quad k \in \mathbb{N} .
$$


Из (18), (20), теоремы 3 при $p=m+1$ и тождества Гильберта имеем

$$
\begin{aligned}
\varphi_{k}^{(m+1)}-\varphi_{\infty}^{(m+1)} & =\frac{1}{2^{1 / 2}}\left(0 ;\left[\frac{1}{\lambda_{k}^{1 / 2}}\left(\mathcal{G}-\lambda_{k}^{(m+1)}\right)^{-1}-i \alpha^{-1 / 2} I\right] \mathcal{Q}\right)^{\tau}= \\
& =\frac{1}{2^{1 / 2}}\left(0 ;\left[I-i \alpha^{-1 / 2} \lambda_{k}^{1 / 2}\left(\mathcal{G}-\lambda_{k}^{(m+1)}\right)\right]\left(\lambda_{k}^{1 / 2} \mathcal{G}-\lambda_{k}^{1 / 2} \lambda_{k}^{(m+1)}\right)^{-1} \mathcal{Q}\right)^{\tau}= \\
& =\lambda_{k}^{1 / 2} \cdot \frac{1}{2^{1 / 2}}\left(0 ;\left[\frac{i \alpha^{-3 / 2}}{2} \sum_{l=1}^{m} \beta_{l} b_{l} I-i \alpha^{-1 / 2} \mathcal{G}+O\left(\lambda_{k}^{1 / 2}\right)\right]\left(\lambda_{k}^{1 / 2} \mathcal{G}-\lambda_{k}^{1 / 2} \lambda_{k}^{(m+1)}\right)^{-1} \mathcal{Q}\right)^{\tau}
\end{aligned}
$$

при $k \rightarrow+\infty$. Аналогичные вычисления справедливы и при $p=m+2$. Таким образом, имеют место неравенства (22) при $p=m+1$ и $p=m+2$. Теперь из (21) и (22) следует утверждение леммы. Лемма доказана.

6. О p-базисности системы корневых элементов оператора $\mathcal{A}$. Следствием лемм 6 и 7 является следующее утверждение.

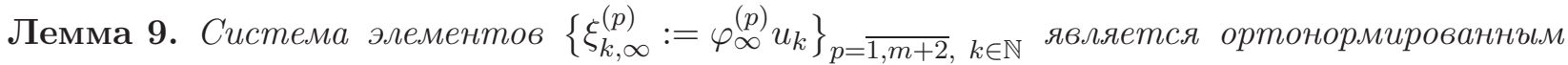
базисом пространства $\mathcal{H}$.

Доказательство. Ортонормированность введенной системы следует из леммы 6 и ортонормированности системы $\left\{u_{k}\right\}_{k=1}^{\infty}$. Покажем, что введенная система полна в $\mathcal{H}$. Пусть существует такой $\xi=\left(v ; v_{0} ; v_{1} ; \ldots ; v_{m}\right)^{\tau} \in \mathcal{H}$, что $\left(\xi_{k, \infty}^{(p)}, \xi\right)_{\mathcal{H}}=0$ при всех $p=\overline{1, m+2}, k \in \mathbb{N}$. Последнее означает, что

$$
M_{\infty}^{\tau}\left(\left(u_{k}, v\right)_{H},\left(u_{k}, v_{0}\right)_{H},\left(u_{k}, v_{1}\right)_{H}, \ldots,\left(u_{k}, v_{m}\right)_{H}\right)^{\tau}=0
$$

при $k \in \mathbb{N}$. Отсюда, из леммы 7 и из полноты системы $\left\{u_{k}\right\}_{k=1}^{\infty}$ в пространстве $H$ тогда получим, что $v=v_{0}=v_{1}=\ldots=v_{m}=0$, т.е. $\xi=0$. Лемма доказана.

C помощью набора матриц $S_{k}(k \in \mathbb{N})$, действующих в $\mathbb{C}^{m+2}$, определим оператор $\mathcal{S}$, действующий в гильбертовом пространстве $\mathcal{H}$, по следующей формуле:

$$
\mathcal{S} \xi:=\sum_{k=1}^{+\infty}\left(\xi_{k, \infty}^{(1)}, \ldots, \xi_{k, \infty}^{(m+2)}\right) S_{k}\left(\begin{array}{c}
\left(\xi, \xi_{k, \infty}^{(1)}\right)_{\mathcal{H}} \\
\vdots \\
\left(\xi, \xi_{k, \infty}^{(m+2)}\right)_{\mathcal{H}}
\end{array}\right):=\sum_{k=1}^{+\infty}\left[\sum_{p=1}^{m+2} \xi_{k, \infty}^{(p)} \sum_{q=1}^{m+2} S_{k}^{p q}\left(\xi, \xi_{k, \infty}^{(q)}\right)_{\mathcal{H}}\right]
$$

и будем писать при этом $\mathcal{S} \leftrightarrow S_{k}$.

Лемма 10. Имеют место следующие утверждения.

1. $\|\mathcal{S}\|_{\mathcal{L}(\mathcal{H})} \leqslant \sup _{k \in \mathbb{N}}\left\|S_{k}\right\|_{\mathcal{L}\left(\mathbb{C}^{m+2}\right)}$.

2. Пусть $\mathcal{S} \in \mathcal{L}(\mathcal{H})$. Если $\mathcal{S} \leftrightarrow S_{k}$, оо $^{*} \leftrightarrow S_{k}^{*}$.

3. Пусть $\mathcal{S}, \mathcal{T} \in \mathcal{L}(\mathcal{H})$, тогда $\mathcal{S} \mathcal{T} \leftrightarrow S_{k} T_{k}$. В частности, $\mathcal{S}^{-1} \leftrightarrow S_{k}^{-1}$.

Доказательство. Лемма доказывается непосредственной проверкой с использованием ортонормированности системы $\left\{\xi_{k, \infty}^{(p)}\right\}_{p=\overline{1, m+2}, k \in \mathbb{N}}$.

Основываясь на доказанных фактах, установим две теоремы: о $p$-базисности специальным образом нормированной системы собственных элементов оператора $\mathcal{A}$ в невырожденном случае и о $p$-базисности системы корневых элементов оператора $\mathcal{A}$ в вырожденном случае.

Теорема 4. Пусть $g_{k}^{\prime}\left(\lambda_{k}^{(p)}\right) \neq 0(p=\overline{1, m+2}, k \in \mathbb{N})$. Тогда система собственных элементов $\left\{\xi_{k}^{(p)}:=\varphi_{k}^{(p)} u_{k}\right\}_{p=\overline{1, m+2}, k \in \mathbb{N}}$ оператора $\mathcal{A}$ образует базис Рисса в $\mathcal{H}$. Если $A^{-1} \in \mathfrak{S}_{q}(H)$ при некотором $q>0$, то система собственных элементов оператора $\mathcal{A}$ образует р-базис в гильбертовом пространстве $\mathcal{H}$ при $p \geqslant 2 q$. 
Доказательство. Положим $\mathcal{S} \leftrightarrow S_{k}:=M_{\infty}^{*} M_{k}$ и покажем, что $\mathcal{S} \xi_{l, \infty}^{(q)}=\xi_{l}^{(q)}$ при $q=\overline{1, m+2}, l \in \mathbb{N}$. Учитывая, что $S_{k}^{p q}={\overline{\left(\varphi_{\infty}^{(p)}, \varphi_{k}^{(q)}\right)_{\mathbb{C}^{m+2}}}}$ и $\left(\xi_{l}^{(q)}, \xi_{k, \infty}^{(p)}\right)_{\mathcal{H}}=0$ при $l \neq k$, вычислим

$$
\begin{aligned}
& \mathcal{S} \xi_{l, \infty}^{(q)}=\left(\xi_{l, \infty}^{(1)}, \ldots, \xi_{l, \infty}^{(m+2)}\right) M_{\infty}^{*} M_{l}\left(0, \ldots, 0,1_{q}, 0, \ldots, 0\right)^{\tau}=
\end{aligned}
$$

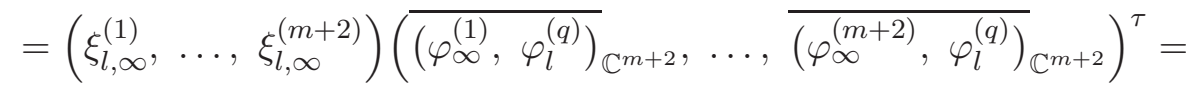

$$
\begin{aligned}
& =\sum_{p=1}^{m+2} \overline{\left(\varphi_{\infty}^{(p)}, \varphi_{l}^{(q)}\right)_{\mathbb{C}^{m+2}}} \xi_{l, \infty}^{(p)}=\sum_{p=1}^{m+2}\left(\varphi_{l}^{(q)}, \varphi_{\infty}^{(p)}\right)_{\mathbb{C}^{m+2}} \xi_{l, \infty}^{(p)}= \\
& =\sum_{p=1}^{m+2}\left(\xi_{l}^{(q)}, \xi_{l, \infty}^{(p)}\right)_{\mathcal{H}} \xi_{l, \infty}^{(p)}=\sum_{k=1}^{+\infty} \sum_{p=1}^{m+2}\left(\xi_{l}^{(q)}, \xi_{k, \infty}^{(p)}\right)_{\mathcal{H}} \xi_{k, \infty}^{(p)}=\xi_{l}^{(q)} .
\end{aligned}
$$

Из леммы 10, условия $g_{k}^{\prime}\left(\lambda_{k}^{(p)}\right) \neq 0(p=\overline{1, m+2}, k \in \mathbb{N})$, лемм 5 и 7 следует, что оператор $\mathcal{S}$ непрерывно обратим: $\mathcal{S}^{-1} \in \mathcal{L}(\mathcal{H})$. Отсюда и из леммы 9 тогда следует, что система элементов $\left\{\xi_{k}^{(p)}\right\}_{p=1, m+2}, k \in \mathbb{N}-$ базис Рисса пространства $\mathcal{H}$. Для доказательства теоремы остается показать, что $\mathcal{S}=\mathcal{I}+\mathcal{T}$, где $\mathcal{T} \in \mathfrak{S}_{p}(\mathcal{H})$ при $p \geqslant 2 q$.

Положим $T_{k}:=M_{k}-M_{\infty}$; тогда с учетом лемм 7 и 10 получим

$$
\begin{gathered}
\mathcal{S} \leftrightarrow M_{\infty}^{*} M_{k}=M_{\infty}^{*}\left(M_{\infty}+T_{k}\right)=I+M_{\infty}^{*} T_{k} \leftrightarrow \mathcal{I}+\mathcal{T}, \\
\mathcal{T}^{*} \mathcal{T} \leftrightarrow\left(T_{k}^{*} M_{\infty}\right)\left(M_{\infty}^{*} T_{k}\right)=T_{k}^{*} T_{k} .
\end{gathered}
$$

Обозначим через $\lambda_{k}\left(\left(\mathcal{T}^{*} \mathcal{T}\right)^{1 / 2}\right)$ и $\lambda_{k}\left(\left(T^{*} T\right)^{1 / 2}\right)$ собственные значения оператора $\left(\mathcal{T}^{*} \mathcal{T}\right)^{1 / 2}$ и матрицы $\left(T^{*} T\right)^{1 / 2}$ соответственно, занумерованные в порядке убывания и с учетом кратности. Тогда из последних соотношений и леммы 9 получим, что

$$
\begin{gathered}
\sum_{r=1}^{+\infty} \lambda_{r}^{p}\left(\left(\mathcal{T}^{*} \mathcal{T}\right)^{1 / 2}\right)=\sum_{k=1}^{+\infty} \sum_{l=1}^{m+2} \lambda_{l}^{p}\left(\left(T_{k}^{*} T_{k}\right)^{1 / 2}\right)=\sum_{k=1}^{+\infty} \sum_{l=1}^{m+2}\left[\lambda_{l}\left(T_{k}^{*} T_{k}\right)\right]^{p / 2} \leqslant \\
\leqslant(m+2) \sum_{k=1}^{+\infty}\left[\lambda_{\max }\left(T_{k}^{*} T_{k}\right)\right]^{p / 2}=(m+2) \sum_{k=1}^{+\infty}\left\|T_{k}^{*} T_{k}\right\|_{\mathcal{L}\left(\mathbb{C}^{m+2}\right)}^{p / 2} \leqslant \\
\leqslant(m+2) \sum_{k=1}^{+\infty}\left\|T_{k}\right\|_{\mathcal{L}\left(\mathbb{C}^{m+2}\right)}^{p} \leqslant(m+2) C^{p} \sum_{k=1}^{+\infty}\left[\lambda_{k}\left(A^{-1}\right)\right]^{p / 2}<+\infty
\end{gathered}
$$

при $p / 2 \geqslant q$, так как $A^{-1} \in \mathfrak{S}_{q}(H)$. Следовательно, $\mathcal{T} \in \mathfrak{S}_{p}(\mathcal{H})$ при $p \geqslant 2 q$. Теорема доказана.

Рассмотрим теперь ситуацию, когда при некотором $k \in \mathbb{N}$ уравнение $g_{k}(\lambda)=0$ имеет кратный корень. В этом случае может быть один или два двукратных корня либо один трехкратный корень.

Разберем случай двукратного корня. В этом случае при некоторых $k \in \mathbb{N}$ (таких номеров конечное количество) будет либо $\lambda_{k}^{(m+1)}=\lambda_{k}^{(m+2)} \in \mathbb{R}$, либо $\lambda_{k}^{\left(p_{1}\right)}=\lambda_{k}^{(m+1)}, \lambda_{k}^{\left(p_{2}\right)}=\lambda_{k}^{(m+2)}$ при некоторых $p_{1}, p_{2} \in\{1, \ldots, m\}$. Не ограничивая общности, предположим первую ситуацию. Пусть $u-$ это первый присоединенный элемент к собственному элементу $u_{k}$ пучка $L(\lambda)$ в точке $\lambda_{k}^{(m+2)}$ (см. определение 2). Тогда $L^{\prime}\left(\lambda_{k}^{(m+2)}\right) u_{k}=g_{k}^{\prime}\left(\lambda_{k}^{(m+2)}\right) u_{k}=0$ и

$$
L^{\prime}\left(\lambda_{k}^{(m+2)}\right) u_{k}+L\left(\lambda_{k}^{(m+2)}\right) u=g_{k}^{\prime}\left(\lambda_{k}^{(m+2)}\right) u_{k}+L\left(\lambda_{k}^{(m+2)}\right) u=L\left(\lambda_{k}^{(m+2)}\right) u=0 .
$$

Таким образом, в качестве первого присоединенного к $u_{k}$ элемента можно взять элемент $u_{k}$.

Пусть $\xi_{k 0}^{(m+2)}=\left(\lambda_{k}^{1 / 2} u_{k} ;\left(\mathcal{G}-\lambda_{k}^{(m+2)}\right)^{-1} \mathcal{Q} u_{k}\right)^{\tau}$ - собственный элемент оператора $\mathcal{A}$, отвечающий собственному значению $\lambda_{k}^{(m+2)}$ (см. теорему 2). Вычислим в соответствии с теоремой 2 присоединенный элемент $\eta_{1}$ оператора $\mathcal{A}$. Поскольку присоединенный элемент определяется с точностью 
до собственного элемента, то можно считать, что

$$
\xi_{k 1}^{(m+2)}=\eta_{1}-\xi_{k 0}^{(m+2)}=\left(0,\left(\mathcal{G}-\lambda_{k}^{(m+2)}\right)^{-2} \mathcal{Q} u_{k}\right)^{\tau}
$$

Следовательно, оператор $\mathcal{A}$ имеет следующую цепочку из собственного и присоединенного к нему элемента:

$$
\begin{aligned}
& \xi_{k 0}^{(m+2)}=\left(\lambda_{k}^{1 / 2},\left(\mathcal{G}-\lambda_{k}^{(m+2)}\right)^{-1} \mathcal{Q}\right)^{\tau} u_{k}=: \varphi_{k 0}^{(m+2)} u_{k}, \\
& \xi_{k 1}^{(m+2)}=\left(0,\left(\mathcal{G}-\lambda_{k}^{(m+2)}\right)^{-2} \mathcal{Q}\right)^{\tau} u_{k}=: \varphi_{k 1}^{(m+2)} u_{k} .
\end{aligned}
$$

Разберем теперь ситуацию, когда при некотором $k \in \mathbb{N}$ уравнение $g_{k}(\lambda)=0$ имеет трехкратный корень. В этом случае при некотором $p \in\{1, \ldots, m\}$ будет $\lambda_{k}^{(p)}=\lambda_{k}^{(m+1)}=\lambda_{k}^{(m+2)} \in \mathbb{R}$. Пусть $u-$ это второй присоединенный элемент к собственному элементу $u_{k}$ пучка $L(\lambda)$ в точке $\lambda_{k}^{(p)}$. Тогда

$$
\begin{gathered}
L^{\prime \prime}\left(\lambda_{k}^{(p)}\right) u_{k}=g_{k}^{\prime \prime}\left(\lambda_{k}^{(p)}\right) u_{k}=g_{\infty}^{\prime \prime}\left(\lambda_{k}^{(p)}\right) u_{k}=0, \quad L^{\prime}\left(\lambda_{k}^{(p)}\right) u_{k}=g_{k}^{\prime}\left(\lambda_{k}^{(p)}\right) u_{k}=0 \\
\frac{1}{2} L^{\prime \prime}\left(\lambda_{k}^{(p)}\right) u_{k}+L^{\prime}\left(\lambda_{k}^{(p)}\right) u_{k}+L\left(\lambda_{k}^{(p)}\right) u=\frac{1}{2} g_{\infty}^{\prime \prime}\left(\lambda_{k}^{(p)}\right) u_{k}+g_{k}^{\prime}\left(\lambda_{k}^{(p)}\right) u_{k}+L\left(\lambda_{k}^{(p)}\right) u=L\left(\lambda_{k}^{(p)}\right) u=0 .
\end{gathered}
$$

Таким образом, в качестве второго присоединенного к $u_{k}$ элемента можно взять элемент $u_{k}$.

Вычислим, в соответствии с теоремой 2, первый $\eta_{1}$ и второй $\eta_{2}$ присоединенные элементы оператора $\mathcal{A}$. Легко проверить, что цепочкой из собственного и присоединенных к нему элементов будет также $\xi_{k 0}^{(p)}, \xi_{k 1}^{(p)}:=\eta_{1}-\xi_{k 0}^{(p)}, x i_{k 2}^{(p)}:=\eta_{2}-\eta_{1}$. Таким образом, имеем

$$
\begin{aligned}
& \xi_{k 0}^{(p)}=\left(\lambda_{k}^{1 / 2},\left(\mathcal{G}-\lambda_{k}^{(p)}\right)^{-1} \mathcal{Q}\right)^{\tau} u_{k}=: \varphi_{k 0}^{(p)} u_{k}, \\
& \xi_{k 1}^{(p)}=\left(0,\left(\mathcal{G}-\lambda_{k}^{(p)}\right)^{-2} \mathcal{Q}\right)^{\tau} u_{k}=: \varphi_{k 1}^{(p)} u_{k}, \\
& \xi_{k 2}^{(p)}=\left(0 ;\left(\mathcal{G}-\lambda_{k}^{(p)}\right)^{-3} \mathcal{Q}\right)^{\tau} u_{k}=: \varphi_{k 2}^{(p)} u_{k} .
\end{aligned}
$$

Далее будем считать, что система корневых элементов оператора $\mathcal{A}$ нормируется следующим образом: если собственный элемент не имеет присоединенного, то он выбирается по формуле из теоремы 2; если собственный элемент имеет один или два присоединенных элемента, то соответствующая цепочка выбирается по формуле (23) или (24) соответственно.

Отметим, что собственных элементов оператора $\mathcal{A}$, имеющих один или два присоединенных элемента может быть лишь конечное количество.

Теорема 5. Система корневых элементов оператора $\mathcal{A}$, нормированных специалъным образом, образует базис Рисса в пространстве $\mathcal{H}$. Если $A^{-1} \in \mathfrak{S}_{q}(H)$ при некотором $q>0$, то система корневых элементов оператора $\mathcal{A}$ образует $p$-базис в пространстве $\mathcal{H}$ при $p \geqslant 2 q$.

Доказательство. Покажем сначала, что система корневых элементов оператора $\mathcal{A}$ полна в $\mathcal{H}$. Рассмотрим для простоты ситуацию, когда у оператора $\mathcal{A}$ есть одно собственное значение $\lambda_{s}^{(p)}$, которому отвечает цепочка из собственного и одного или двух присоединенных элементов. Проведем доказательство в несколько этапов.

1. Пусть собственному значению $\lambda_{s}^{(m+2)}$ оператора $\mathcal{A}$ отвечает цепочка из собственного и присоединенного к нему элемента, определяемых по формулам (23). Предположим, что рассматриваемая система не полна в $\mathcal{H}$ и существует такой $\xi=\left(v, v_{0}, v_{1}, \ldots, v_{m}\right)^{\tau} \in \mathcal{H}$, что

$$
\begin{aligned}
\left(\xi_{k}^{(p)}, \xi\right)_{\mathcal{H}} & =0, \quad p=\overline{1, m+2}, \quad k=1,2, \ldots, s-1, s+1, \ldots, \\
\left(\xi_{s}^{(p)}, \xi\right)_{\mathcal{H}} & =0, \quad p=\overline{1, m}, \quad\left(\xi_{s 0}^{(m+2)}, \xi\right)_{\mathcal{H}}=0, \quad\left(\xi_{s 1}^{(m+2)}, \xi\right)_{\mathcal{H}}=0 .
\end{aligned}
$$

Первая строчка в (25) означает, что

$$
M_{k}^{\tau}\left(\left(u_{k}, v\right)_{H},\left(u_{k}, v_{0}\right)_{H},\left(u_{k}, v_{1}\right)_{H}, \ldots,\left(u_{k}, v_{m}\right)_{H}\right)^{\tau}=0
$$

при $k=1,2, \ldots, s-1, s+1, \ldots$ (см. лемму 5). Отсюда следует, что

$$
\left(u_{k}, v\right)_{H}=\left(u_{k}, v_{0}\right)_{H}=\ldots=\left(u_{k}, v_{m}\right)_{H}=0, \quad k \in \mathbb{N} \backslash\{s\} .
$$


Вторая строчка в (25) означает, что

$$
M_{s, 1}^{\tau}\left(\left(u_{s}, v\right)_{H},\left(u_{s}, v_{0}\right)_{H},\left(u_{s}, v_{1}\right)_{H}, \ldots,\left(u_{s}, v_{m}\right)_{H}\right)^{\tau}=0,
$$

где $M_{s, 1}=M_{s, 1}\left(\varphi_{s}^{(1)}, \varphi_{s}^{(2)}, \ldots, \varphi_{s}^{(m)}, \varphi_{s 0}^{(m+2)}, \varphi_{s 1}^{(m+2)}\right)$ - матрица, столбцами которой являются соответствующие векторы. Покажем, что $\operatorname{det} M_{s, 1} \neq 0$; тогда из последней системы, соотношений (26) и полноты в $H$ системы $\left\{u_{k}\right\}_{k=1}^{\infty}$ получим, что $v=v_{0}=v_{1}=\ldots=v_{m}=0$, т.е. $\xi=0$.

Пусть, как и в лемме 4 and $J=\operatorname{diag}(1,-I)$. Несложно проверить, что

$$
\begin{gathered}
\left(J \varphi_{s 0}^{(m+2)}, \varphi_{s 0}^{(m+2)}\right)_{\mathbb{C}^{m+2}}=0, \quad\left(J \varphi_{s 0}^{(m+2)}, \varphi_{s 1}^{(m+2)}\right)_{\mathbb{C}^{m+2}}=-\frac{1}{2} g_{s}^{\prime \prime}\left(\lambda_{s}^{(m+2)}\right) \neq 0 \\
\left(J \varphi_{s 1}^{(m+2)}, \varphi_{s 1}^{(m+2)}\right)_{\mathbb{C}^{m+2}}=-\frac{1}{3 !} g_{s}^{\prime \prime \prime}\left(\lambda_{s}^{(m+2)}\right) .
\end{gathered}
$$

Для дальнейших вычислений понадобится формула, которая может быть получена последовательным дифференцированием тождества Гильберта:

$$
(\mathcal{G}-\lambda)^{-n}(\mathcal{G}-\mu)^{-1}=\frac{1}{(\mu-\lambda)^{n}}(\mathcal{G}-\mu)^{-1}-\sum_{k=0}^{n-1} \frac{1}{(\mu-\lambda)^{k+1}}(\mathcal{G}-\lambda)^{-(n-k)}, \quad \mu, \lambda \in \rho(\mathcal{G}), \quad n \in \mathbb{N} .
$$

Используя соотношения $g_{s}\left(\lambda_{s}^{(p)}\right)=0(p=\overline{1, m})($ см. $(17)), g_{s}\left(\lambda_{s}^{(m+2)}\right)=g_{s}^{\prime}\left(\lambda_{s}^{(m+2)}\right)=0$, формулы (28) при $n=2$, можно найти, что при всех $p=\overline{1, m}$

$$
\begin{aligned}
&\left(J \varphi_{s}^{(p)}, \varphi_{s 1}^{(m+2)}\right)_{\mathbb{C}^{m+2}}=-R_{s, p}\left(\left(\mathcal{G}-\lambda_{s}^{(p)}\right)^{-1} \mathcal{Q}\left(\mathcal{G}-\lambda_{s}^{(m+2)}\right)^{-2} \mathcal{Q}\right)_{\mathbb{C}^{m+1}}= \\
&=-R_{s, p} \mathcal{Q}^{*}\left(\mathcal{G}-\lambda_{s}^{(m+2)}\right)^{-2}\left(\mathcal{G}-\lambda_{s}^{(p)}\right)^{-1} \mathcal{Q}= \\
&=-R_{s, p} \mathcal{Q}^{*}\left[\frac{\left(\mathcal{G}-\lambda_{s}^{(p)}\right)^{-1}}{\left(\lambda_{s}^{(p)}-\lambda_{s}^{(m+2)}\right)^{2}}-\frac{\left(\mathcal{G}-\lambda_{s}^{(m+2)}\right)^{-2}}{\lambda_{s}^{(p)}-\lambda_{s}^{(m+2)}}-\frac{\left(\mathcal{G}-\lambda_{s}^{(m+2)}\right)^{-1}}{\left(\lambda_{s}^{(p)}-\lambda_{s}^{(m+2)}\right)^{2}}\right] \mathcal{Q}= \\
&=-R_{s, p}\left[\frac{\lambda_{s}^{(p)} \lambda_{k}}{\left(\lambda_{s}^{(p)}-\lambda_{s}^{(m+2)}\right)^{2}}-\frac{\lambda_{k}}{\lambda_{s}^{(p)}-\lambda_{s}^{(m+2)}}-\frac{\lambda_{s}^{(m+2)} \lambda_{k}}{\left(\lambda_{s}^{(p)}-\lambda_{s}^{(m+2)}\right)^{2}}\right]=0 .
\end{aligned}
$$

Из (27) и (29) найдем, что

$$
\begin{gathered}
M_{s, 1}^{\tau} J M_{s, 1}=\left(\begin{array}{ccccc}
-g_{s}^{\prime}\left(\lambda_{s}^{(1)}\right) R_{s, 1}^{2} & 0 & \ldots & 0 & 0 \\
0 & -g_{s}^{\prime}\left(\lambda_{s}^{(2)}\right) R_{s, 2}^{2} & \ldots & 0 & 0 \\
\vdots & \vdots & \ddots & \vdots & \vdots \\
0 & 0 & \ldots & 0 & -\frac{1}{2 !} g_{s}^{\prime \prime}\left(\lambda_{s}^{(m+2)}\right) \\
0 & 0 & \ldots & -\frac{1}{2 !} g_{s}^{\prime \prime}\left(\lambda_{s}^{(m+2)}\right) & -\frac{1}{3 !} g_{s}^{\prime \prime \prime}\left(\lambda_{s}^{(m+2)}\right)
\end{array}\right), \\
(-1)^{m+1}\left(\operatorname{det} M_{s, 1}\right)^{2}=\operatorname{det} M_{s, 1}^{\tau} J M_{s, 1}=(-1)^{m+1}\left[\frac{1}{2 !} g_{s}^{\prime \prime}\left(\lambda_{s}^{(m+2)}\right)\right]^{2} \prod_{p=1}^{m} g_{s}^{\prime}\left(\lambda_{s}^{(p)}\right) R_{s, p}^{2} \neq 0 .
\end{gathered}
$$

Следовательно, $\operatorname{det} M_{s, 1} \neq 0$.

2. Пусть теперь собственному значению $\lambda_{s}^{(p)}$ оператора $\mathcal{A}$ отвечает цепочка из собственного и двух присоединенных элементов, определяемых по формулам (24). Не ограничивая общности можно считать, что $p=m$. Предположим, что система корневых элементов оператора $\mathcal{A}$ не полна в $\mathcal{H}$ и существует такой $\xi=\left(v, v_{0}, v_{1}, \ldots, v_{m}\right)^{\tau} \in \mathcal{H}$, что

$$
\begin{aligned}
& \left(\xi_{k}^{(p)}, \xi\right)_{\mathcal{H}}=0, \quad p=\overline{1, m+2}, \quad k=1,2, \ldots, s-1, s+1, \ldots, \\
& \left(\xi_{s}^{(p)}, \xi\right)_{\mathcal{H}}=0, \quad p=\overline{1, m-1}, \quad\left(\xi_{s 0}^{(m)}, \xi\right)_{\mathcal{H}}=0, \quad\left(\xi_{s 1}^{(m)}, \xi\right)_{\mathcal{H}}=0, \quad\left(\xi_{s 2}^{(m)}, \xi\right)_{\mathcal{H}}=0 .
\end{aligned}
$$

Первая строка в (30), как и выше, влечет (26); вторая строка означает, что

$$
M_{s, 2}^{\tau}\left(\left(u_{s}, v\right)_{H},\left(u_{s}, v_{0}\right)_{H},\left(u_{s}, v_{1}\right)_{H}, \ldots,\left(u_{s}, v_{m}\right)_{H}\right)^{\tau}=0,
$$


где $M_{s, 2}=M_{s, 2}\left(\varphi_{s}^{(1)}, \ldots, \varphi_{s}^{(m-1)}, \varphi_{s 0}^{(m)}, \varphi_{s 1}^{(m)}, \varphi_{s 2}^{(m)}\right)$ - матрица, столбцами которой являются соответствующие векторы. Покажем, что $\operatorname{det} M_{s, 2} \neq 0$; тогда из последней системы, соотношений (26) и полноты в $H$ системы $\left\{u_{k}\right\}_{k=1}^{\infty}$ получим, как и выше, что $v=v_{0}=v_{1}=\ldots=v_{m}=0$ и, значит, $\xi=0$.

Несложно проверить, что

$$
\begin{gathered}
\left(J \varphi_{s 0}^{(m)}, \varphi_{s 0}^{(m)}\right)_{\mathbb{C}^{m+2}}=0, \quad\left(J \varphi_{s 0}^{(m)}, \varphi_{s 1}^{(m)}\right)_{\mathbb{C}^{m+2}}=0 \\
\left(J \varphi_{s 0}^{(m)}, \varphi_{s 2}^{(m)}\right)_{\mathbb{C}^{m+2}}=\left(J \varphi_{s 1}^{(m)}, \varphi_{s 1}^{(m)}\right)_{\mathbb{C}^{m+2}}=-\frac{1}{3 !} g_{s}^{\prime \prime \prime}\left(\lambda_{s}^{(m)}\right) \neq 0, \\
\left(J \varphi_{s 1}^{(m)}, \varphi_{s 2}^{(m)}\right)_{\mathbb{C}^{m+2}}=-\frac{1}{4 !} g_{s}^{(4)}\left(\lambda_{s}^{(m)}\right), \quad\left(J \varphi_{s 2}^{(m)}, \varphi_{s 2}^{(m)}\right)_{\mathbb{C}^{m+2}}=-\frac{1}{5 !} g_{s}^{(5)}\left(\lambda_{s}^{(m)}\right), \\
\left(J \varphi_{s}^{(p)}, \varphi_{s 1}^{(m)}\right)_{\mathbb{C}^{m+2}}=0, \quad p=1,2, \ldots, m-1 .
\end{gathered}
$$

Здесь последнее соотношение выводится также как в (29). Далее, с использованием соотношений

$$
g_{s}\left(\lambda_{s}^{(p)}\right)=0, \quad(p=\overline{1, m-1}), \quad g_{s}\left(\lambda_{s}^{(m)}\right)=g_{s}^{\prime}\left(\lambda_{s}^{(m)}\right)=g_{s}^{\prime \prime}\left(\lambda_{s}^{(m)}\right)=0
$$

(см. (17)), формулы (28) при $n=3$ можно найти, что при всех $p=\overline{1, m-1}$

$$
\begin{aligned}
&\left(J \varphi_{s}^{(p)},\right.\left.\varphi_{s 2}^{(m)}\right)_{\mathbb{C}^{m+2}}= \\
&=-R_{s, p}\left(\left(\mathcal{G}-\lambda_{s}^{(p)}\right)^{-1} \mathcal{Q},\left(\mathcal{G}-\lambda_{s}^{(m)}\right)^{-3} \mathcal{Q}\right)_{\mathbb{C}^{m+1}}=-R_{s, p} \mathcal{Q}^{*}\left(\mathcal{G}-\lambda_{s}^{(m)}\right)^{-3}\left(\mathcal{G}-\lambda_{s}^{(p)}\right)^{-1} \mathcal{Q}= \\
&=-R_{s, p} \mathcal{Q}^{*}\left[\frac{\left(\mathcal{G}-\lambda_{s}^{(p)}\right)^{-1}}{\left(\lambda_{s}^{(p)}-\lambda_{s}^{(m)}\right)^{3}}-\frac{\left(\mathcal{G}-\lambda_{s}^{(m)}\right)^{-3}}{\lambda_{s}^{(p)}-\lambda_{s}^{(m)}}-\frac{\left(\mathcal{G}-\lambda_{s}^{(m)}\right)^{-2}}{\left(\lambda_{s}^{(p)}-\lambda_{s}^{(m)}\right)^{2}}-\frac{\left(\mathcal{G}-\lambda_{s}^{(m)}\right)^{-1}}{\left(\lambda_{s}^{(p)}-\lambda_{s}^{(m)}\right)^{3}}\right] \mathcal{Q}= \\
& \quad=-R_{s, p}\left[\frac{\lambda_{s}^{(p)} \lambda_{k}}{\left(\lambda_{s}^{(p)}-\lambda_{s}^{(m)}\right)^{3}}-\frac{\lambda_{k}}{\left(\lambda_{s}^{(p)}-\lambda_{s}^{(m)}\right)^{2}}-\frac{\lambda_{s}^{(m)} \lambda_{k}}{\left(\lambda_{s}^{(p)}-\lambda_{s}^{(m+2)}\right)^{3}}\right]=0 .
\end{aligned}
$$

Из (31) и (32) найдем, что

$$
\begin{gathered}
M_{s, 2}^{\tau} J M_{s, 2}=\left(\begin{array}{cccccc}
-g_{s}^{\prime}\left(\lambda_{s}^{(1)}\right) R_{s, 1}^{2} & 0 & \ldots & 0 & 0 & 0 \\
0 & -g_{s}^{\prime}\left(\lambda_{s}^{(2)}\right) R_{s, 2}^{2} & \ldots & 0 & 0 & 0 \\
\vdots & \vdots & \ddots & \vdots & \vdots & \vdots \\
0 & 0 & \ldots & 0 & 0 & -\frac{1}{3 !} g_{s}^{\prime \prime \prime}\left(\lambda_{s}^{(m)}\right) \\
0 & 0 & \ldots & 0 & -\frac{1}{3 !} g_{s}^{\prime \prime \prime}\left(\lambda_{s}^{(m)}\right) & -\frac{1}{4 !} g_{s}^{(4)}\left(\lambda_{s}^{(m)}\right) \\
0 & 0 & \ldots & -\frac{1}{3 !} g_{s}^{\prime \prime \prime}\left(\lambda_{s}^{(m)}\right) & -\frac{1}{4 !} g_{s}^{(4)}\left(\lambda_{s}^{(m)}\right) & -\frac{1}{5 !} g_{s}^{(5)}\left(\lambda_{s}^{(m)}\right)
\end{array}\right) \\
(-1)^{m+1}\left(\operatorname{det} M_{s, 2}\right)^{2}=\operatorname{det} M_{s, 2}^{\tau} J M_{s, 2}=(-1)^{m-1}\left[\frac{1}{3 !} g_{s}^{\prime \prime \prime}\left(\lambda_{s}^{(m)}\right)\right] \prod_{p=1}^{3 m-1} g_{s}^{\prime}\left(\lambda_{s}^{(p)}\right) R_{s, p}^{2} \neq 0 .
\end{gathered}
$$

Таким образом, $\operatorname{det} M_{s, 2} \neq 0$, и система корневых элементов оператора $\mathcal{A}$ полна в $\mathcal{H}$.

3. Построим теперь, как и в теореме 4 , оператор $\mathcal{S} \leftrightarrow S_{k}:=M_{\infty}^{*} M_{k}$ с заменой вырожденных матриц $M_{s}$ на какие либо невырожденные. При этом оператор $\mathcal{S}$ будет непрерывно обратим и попрежнему представим в виде $\mathcal{S}=\mathcal{I}+\mathcal{T}$, где $\mathcal{T} \in \mathfrak{S}_{p}(\mathcal{H})(p \geqslant 2 q)$, так как вырожденных матриц $M_{s}$ может быть лишь конечное количество. Таким образом, система $\left\{\mathcal{S} \xi_{k, \infty}^{(p)}\right\}_{p=\overline{1, m+2}, k \in \mathbb{N}}$ есть $p$ базис $(p \geqslant 2 q)$ пространства $\mathcal{H}$, который отличается от системы специальным образом нормированных корневых элементов оператора $\mathcal{A}$ лишь на конечное количество элементов. Отсюда следует, что система корневых элементов оператора $\mathcal{A}$, учитывая ее полноту, есть также $p$-базис $(p \geqslant 2 q)$ пространства $\mathcal{H}$. Теорема доказана.

В качестве следствия из леммы 4 и теоремы 4 (невырожденный случай) сформулируем следующее утверждение. 
Теорема 6. Пусть $A^{-1} \in \mathfrak{S}_{q}(H)(q>0) u g_{k}^{\prime}\left(\lambda_{k}^{(p)}\right) \neq 0(p=\overline{1, m+2}, k \in \mathbb{N})$. Тогда система

$$
\begin{gathered}
\xi_{k}^{(p)}:=R_{k, p}\left(\lambda_{k}^{1 / 2}\left(A^{-1}\right),\left(\mathcal{G}-\lambda_{k}^{(p)}\right)^{-1} \mathcal{Q}\right)^{\tau} u_{k}, \quad p=\overline{1, m+2}, \quad k \in \mathbb{N}, \\
R_{k, p}:= \begin{cases}{\left[g_{\infty}^{\prime}\left(\gamma_{p}\right)\right]^{-1 / 2},} & p=\overline{1, m}, \quad k \in \mathbb{N}, \\
{\left[2 \lambda_{k}\left(A^{-1}\right)\right]^{-1 / 2},} & p=m+1, m+2, \quad k \in \mathbb{N},\end{cases}
\end{gathered}
$$

собственных элементов оператора $\mathcal{A}$ образует р-базис пространства $\mathcal{H}$ при $p \geqslant 2 q$, согласно теореме 4, и имеет следующую биортогональную систему:

$$
\zeta_{k}^{(p)}:=-\left[g_{k}^{\prime}\left(\overline{\lambda_{k}^{(p)}}\right) R_{k, p}\right]^{-1}\left(\lambda_{k}^{1 / 2}\left(A^{-1}\right),-\left(\mathcal{G}-\overline{\lambda_{k}^{(p)}}\right)^{-1} \mathcal{Q}\right)^{\tau} u_{k}, \quad p=\overline{1, m+2}, \quad k \in \mathbb{N} .
$$

Доказательство. Теорема доказывается непосредственной проверкой.

7. Представление решения интегро-дифференциального уравнения. Предположим, что $A^{-1} \in \mathfrak{S}_{q}(H)(q>0)$ и собственные элементы оператора $\mathcal{A}$ не имеют присоединенных. Пусть $u^{0} \in \mathcal{D}(A), u^{1} \in \mathcal{D}\left(A^{1 / 2}\right)$, а функция $f(t)$ удовлетворяет условиям теоремы 1 . Тогда по теореме 1 задача Коши (1) имеет единственное сильное решение $u(t)$, а построенная по функции $u(t)$ функция $\xi(t)$ - сильное решение задачи Коши (4) (см. замечание 1). С использованием теоремы 6 это решение $\xi(t)$ представимо в следующем виде:

$$
\xi(t)=\mathcal{U}(t) \xi^{0}+\int_{0}^{t} \mathcal{U}(t-s) \mathcal{F}(s) d s, \quad \mathcal{U}(t) \xi^{0}:=\sum_{k=1}^{+\infty} \sum_{p=1}^{m+2} e^{-\lambda_{k}^{(p)} t}\left(\xi^{0}, \zeta_{k}^{(p)}\right)_{\mathcal{H}} \xi_{k}^{(p)} .
$$

Далее, проведя ряд громоздких, но несложных вычислений с использованием в (33) формул из теоремы 6 , формул для $\xi^{0}, \mathcal{F}(t), \xi(t)$ и связи $u(t)=\beta_{0}^{-1 / 2} A^{-1 / 2} v_{0}(t)$ (см. $(3)$ ), получим формулу для решения задачи Коши (1):

$$
u(t)=\mathcal{C}(t) u^{0}+\mathcal{S}(t) u^{1}+\int_{0}^{t} \mathcal{S}(t-s) f(s) d s
$$

где

$$
\begin{aligned}
\mathcal{C}(t) u^{0} & :=\sum_{k=1}^{+\infty} \sum_{p=1}^{m+2} \frac{\lambda_{k}\left(A^{-1}\right) e^{-\lambda_{k}^{(p)}} t}{\lambda_{k}^{(p)} g_{k}^{\prime}\left(\lambda_{k}^{(p)}\right)}\left[-\lambda_{k}^{(p)} \lambda_{k}\left(A^{-1}\right)+\sum_{l=1}^{m} \frac{\alpha_{-l} e^{-\left(b_{l}-\lambda_{k}^{(p)}\right) t}}{b_{l}\left(b_{l}-\lambda_{k}^{(p)}\right)}\right]\left(A u^{0}, u_{k}\right)_{H} u_{k}, \\
\mathcal{S}(t) u^{1} & :=\sum_{k=1}^{+\infty} \sum_{p=1}^{m+2} \frac{\lambda_{k}\left(A^{-1}\right) e^{-\lambda_{k}^{(p)}} t}{\lambda_{k}^{(p)} g_{k}^{\prime}\left(\lambda_{k}^{(p)}\right)} \lambda_{k}^{1 / 2}\left(A^{-1}\right)\left(A^{1 / 2} u^{1}, u_{k}\right)_{H} u_{k} .
\end{aligned}
$$

8. Приложения. Пусть $\Omega \subset \mathbb{R}^{3}$ - ограниченная область с границей $\partial \Omega$ класса $C^{2}, \rho(x)-$ непрерывная положительная и отделенная от нуля функция в $\bar{\Omega}, \mu, \eta$-некоторые положительные константы. Пусть $H:=\boldsymbol{L}_{2}(\Omega, \rho)$ - векторное гильбертово пространство $\boldsymbol{L}_{2}(\Omega)$ с весом $\rho(x)$. Определим в $H$ оператор

$$
A \boldsymbol{u}:=-\frac{1}{\rho(x)}\left(\mu \Delta \boldsymbol{u}+\left(\eta+\frac{\mu}{3}\right) \nabla \operatorname{div} \boldsymbol{u}\right), \quad \mathcal{D}(A):=\left\{\boldsymbol{u} \in \boldsymbol{L}_{2}(\Omega, \rho) \mid \boldsymbol{u} \in \boldsymbol{W}_{2}^{2}(\Omega), \boldsymbol{u}=\mathbf{0} \text { на } \partial \Omega\right\} ;
$$

тогда, как известно, $A^{-1} \in \mathfrak{S}_{p}(H)$ при $p>3 / 2$. В этом случае задача Коши (1) - это задача о малых движениях неоднородного изотропного вязкоупругого тела, занимающего область $\Omega$ и закрепленного на границе $\partial \Omega$, под действием малого поля внешних сил. При этом $\boldsymbol{u}=\boldsymbol{u}(t, x)-$ поле смещений в вязкоупругом теле, $\rho(x)$ - плотность тела, $\mu, \eta$-коэффициенты Ламе, $\alpha_{-l}-$ структурные постоянные, $b_{l}^{-1}$ - времена релаксации в системе. Система корневых элементов оператора $\mathcal{A}$ из (4), согласно теореме 5 , образует $p$-базис в $\mathcal{H}=\bigoplus_{l=1}^{m+2} \boldsymbol{L}_{2}(\Omega, \rho)$ при $p>3$. В невырожденном случае, т.е. когда собственные элементы оператора $\mathcal{A}$ не имеют присоединенных, решение рассматриваемой задачи может быть представлено в виде (34). 
Если самосопряженный положительно определенный оператор $A$ реализован как $A u=-a u^{(2)}$ $(a>0)$ с условиями Дирихле на интервале $(0, L)$, то задача Коши $(1)$ - это задача о колебаниях вязкоупругой струны. Если оператор $A$ реализован как $A u=a u^{(4)}(a>0)$ с подходящими условиями на интервале $(0, L)$, то задача Коши $(1)$ - это задача о поперечных колебаниях вязкоупругой балки. Если оператор $A$ реализован как $A u=a \Delta^{2} u(a>0)$ в ограниченной области $\Omega \subset \mathbb{R}^{2}$ с условиями жесткого защемления на достаточно гладкой границе $\partial \Omega$, то задача Коши $(1)$ - это задача о поперечных колебаниях вязкоупругой пластины, жестко закрепленной на границе.

\section{СПИСОК ЛИТЕРАТУРЫ}

1. Власов В. В., Раутиан Н. А. Спектральный анализ функционально-дифференциальных уравнений. - M.: MAKC пресс, 2016.

2. Власов В. В., Раутиан Н. А., Шамаев А. С. Спектральный анализ и корректная разрешимость абстрактных интегродифференциальных уравнений, возникающих в теплофизике и акустике// Совр. мат. Фундам. направл. - 2011. - 39. - С. 36-65.

3. Ильюшин А. А., Победря Б. Е. Основы математической теории термовязкоупругости. - М.: Наука, 1970.

4. Крейн С. Г. Линейные дифференциальные уравнения в банаховом пространстве. - М.: Наука, 1967.

5. Kamo T. Теория возмущений линейных операторов. - М.: Мир, 1972.

6. Маркус A. C. Введение в спектральную теорию полиномиальных операторных пучков. - Кишенев: Штиинца, 1986.

7. Alabau-Boussouria F., Cannarsa P. A general method for proving sharp energy decay rates for memorydissipative evolution equations// C. R. Acad. Sci. Paris. Ser. I. - 2009. - 347, № 15-16. - P. 867-872.

8. Dafermos C. M. Asymptotic stability in viscoelasticity// Arch. Rat. Mech. Anal. — 1970. — 37. P. 297-308.

9. Desch W., Grimmer R., Schappacher W. Some considerations for linear integrodifferential equations// J. Math. Anal. Appl. — 1984. - 104, № 1. - P. 219-234.

10. Eremenko A., Ivanov S. Spectra of the Gurtin-Pipkin-type equations// SIAM J. Math. Anal. — 2011. 43, № 5. - P. 2296-2306.

11. Gurtin M. E., Pipkin A. C. A general theory of heat conduction with finite wave speeds// Arch. Rat. Mech. Anal. - 1968. - 31, № 2. - P. 113-126.

12. Heard M. L. An abstract semilinear hyperbolic Volterra integrodifferential equation// J. Math. Anal. Appl. — 1981. - 80, № 1. - P. 175-202.

13. Kopachevsky N. D., Krein S. G. Operator Approach in Linear Problems of Hydrodynamics. Vol. 2. NonSelf-Adjoint Problems for Viscous Fluids. - Basel-Boston-Berlin: Birkhäuser, 2003.

14. Vlasov V. V., Rautian N. A. On the asymptotic behavior of solutions of integrodifferential equations in a Hilbert space// Differ. Equations. — 2013. — 49, № 6. — P. 718-730.

Закора Дмитрий Александрович

Крымский федеральный университет им. В. И. Вернадского

E-mail: dmitry.zkr@gmail.com 